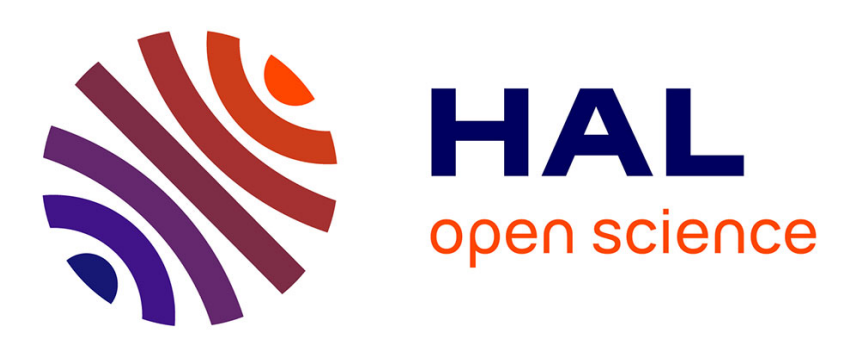

\title{
Un modèle cinétique pour prédire les modifications de structure macromoléculaire du PET pendant son recyclage mécanique
}

\author{
Linda K. Naït-Ali, Anne Bergeret, Xavier Colin
}

\section{- To cite this version:}

Linda K. Naït-Ali, Anne Bergeret, Xavier Colin. Un modèle cinétique pour prédire les modifications de structure macromoléculaire du PET pendant son recyclage mécanique. Matériaux \& Techniques, 2011, 99 (4), pp.373-390. 10.1051/mattech/2011002 . hal-02455984

HAL Id: hal-02455984

https://hal.science/hal-02455984

Submitted on 27 Jan 2020

HAL is a multi-disciplinary open access archive for the deposit and dissemination of scientific research documents, whether they are published or not. The documents may come from teaching and research institutions in France or abroad, or from public or private research centers.
L'archive ouverte pluridisciplinaire HAL, est destinée au dépôt et à la diffusion de documents scientifiques de niveau recherche, publiés ou non, émanant des établissements d'enseignement et de recherche français ou étrangers, des laboratoires publics ou privés. 


\title{
Un modèle cinétique pour prédire les modifications de structure macromoléculaire du PET pendant son recyclage mécanique
}

\author{
L.K. Nait-Ali ${ }^{1}$, A. Bergeret ${ }^{2}$ et X. Colin ${ }^{3}$ \\ 1 Terre Armée Internationale, R\&D Division, 1 bis rue du Petit Clamart, Bâtiment C, BP 135, \\ 78148 Vélizy-Villacoublay, France \\ e-mail : linda.nait-ali@terre-armee.com \\ 2 École des Mines d'Alès, CMGD, 6 avenue de Clavières, 30100 Alès, France \\ e-mail : anne.bergeret@ema.fr \\ 3 Arts et Métiers ParisTech, PIMM (UMR CNRS 8006), 151 boulevard de l'Hôpital, 75013 Paris, France \\ e-mail : xavier.colin@ensam.eu
}

\section{Mots-clés :}

Poly(éthylène térephthalate) ; recyclage mécanique par extrusion; thermooxydation ; coupures et soudures de chaînes ; modélisation cinétique

\begin{abstract}
Résumé - La cavité d'un rhéomètre a été utilisée pour étudier, dans des conditions d'exposition soigneusement contrôlées, les modifications macromoléculaires causées par le recyclage mécanique du PET par extrusion. Des essais de vieillissements isothermes à $280^{\circ} \mathrm{C}$, à des pressions partielles d'oxygène constantes ou variables (entre $0 \%$ et $21 \%$ de la pression atmosphérique), ont permis d'établir que deux types de modifications macromoléculaires oxydantes se produisent successivement dans le réacteur d'une extrudeuse. Les coupures de chaînes prédominent dans les zones "fortement oxygénées " (au niveau de l'alimentation et la tête), alors que les soudures de chaînes prédominent dans la zone "sous-oxygénée " (au centre) du réacteur. Ainsi, il apparaît que la prédominance relative de ces deux types de modifications est étroitement liée à la géométrie et aux dimensions de l'extrudeuse (en particulier, à l'aire des sections d'alimentation et de tête, et la longueur de la vis). Un modèle cinétique de vieillissement thermique du PET à l'état fondu a été élaboré pour vérifier ces hypothèses. II décrit de manière satisfaisante l'ensemble des résultats rhéométriques obtenus dans cette étude.
\end{abstract}

\begin{abstract}
A kinetic model for predicting PET macromolecular changes during its mechanical recycling. The rheometer cavity has been used to analyse, in carefully controlled exposure conditions, the macromolecular changes generated by the mechanical recycling of PET during its extrusion. Isothermal ageing tests at $280^{\circ} \mathrm{C}$, under constant or variable oxygen partial pressures (between $0 \%$ and $21 \%$ of the atmospheric pressure), have allowed to establish that two types of oxidative macromolecular changes take place successively in an extruder reactor. Chain scissions predominate in the "highly oxygenated" zones (at the feeder and die), whereas chain welding predominates in "poorly oxygenated" zones (in the middle) of reactor. Thus, it appears that the relative predominance of both types of modifications is sharply linked to the extruder geometry and size (in particular, to the feeder and die diameter, and screw length). A kinetic model of thermal ageing of molten PET has been built to check these assumptions. It describes satisfyingly all the rheometric results obtained in the present study.
\end{abstract}

a valorisation des déchets en poly(éthylène téréphtalate) (PET) est le résultat de la pression sociétale pour réduire la pollution environnementale, mais aussi de la volonté affichée des industriels de développer des sources de matière première moins onéreuses. Actuellement, les déchets proviennent essentiellement du secteur de l'emballage alimentaire : bouteilles d'eau minérale et de boissons gazeuses, barquettes et films de conditionnement, etc. Ils sont réutilisés après régénération (c'est-à-dire triage, broyage, lavage et séchage) pour la réalisation de fibres textiles ou de nouvelles bouteilles.

Une part importante de la littérature est consacrée à l'étude du recyclage mécanique du PET, c'est-à-dire la mise en œuvre à l'état fondu des paillettes issues du recyclage (souvent par extrusion, mais aussi par injection), ce recyclage pouvant être effectué par mélange de 10 à $20 \%$ en masse de matière vierge. La principale raison de cet engouement 
semble être la diversité et la complexité des processus chimiques (ioniques et radicalaires) impliqués dans cette opération, par exemple :

- 1'hydrolyse des groupes ester [1-6] ;

- l'estérification des extrémités de chaînes acide carboxylique et hydroxyle éthylester [1-6] ;

- la transestérification des paires d'extrémités de chaînes hydroxyle éthylester [4], et des extrémités de chaînes hydroxyle éthylester et vinylester [3,5-9];

- la décomposition thermique des groupes ester de l'unité éthylène glycol (i.e. unité monomère) [3,5-22], des unités diéthylène glycol, considérées comme les principales irrégularités structurales (entre 1 et 3,6\% en moles [23]) [8,23-26] et des extrémités de chaînes hydroxyle éthylester $[4,6,8,10,13,14,20,24]$ et vinylester $[6,10,14,16,18,20,26]$;

- la thermo-oxydation des groupes méthylène de l'unité éthylène glycol [3$6,9,11,21]$ et des unités diéthylène glycol $[3,23,25,27]$;

- la décarbonylation des radicaux acyles et la décarboxylation des radicaux carboxyles [15, 16, 18, 27] ;

- la thermo-oxydation des cycles aromatiques [15];

- 1'hydroxylation des cycles aromatiques [15, 16, 19,23, 24, 26, 28] ;

- la condensation des paires d'extrémités de chaînes acide carboxylique $[5,6,10,22$, 24,26] et des extrémités de chaînes acide carboxylique et vinylester $[3,5,6,8,9,14$, $16,24]$;

- la polyaddition des extrémités de chaînes vinylester $[3,6,8,9,18,23]$;

- la polyaddition des cycles aromatiques [20].

Les réactions d'hydrolyse/estérification conduisent à des changements structuraux pouvant être considérés comme réversibles, à condition que la concentration en eau présente dans le polymère soit bien contrôlée. En effet, on sait que si le taux d'humidité dépasse $0,01-0,02 \%$ en masse [3,29], l'hydrolyse des groupes ester conduit à la formation d'extrémités de chaînes acide carboxylique et hydroxyle éthylester, ce qui se traduit par une chute catastrophique de la masse moléculaire avec le temps de séjour dans la machine de mise en œuvre. Le polymère peut être ensuite facilement régénéré par chauffage à l'état sec. Mais, en général, l’hydrolyse est totalement évitée en séchant soigneusement les granulés/paillettes avant mise en œuvre.

Il en est de même pour les réactions de transestérification. En revanche, tous les autres processus chimiques conduisent à des changements structuraux irréversibles, responsables d'un rétrécissement plus ou moins marqué de la fenêtre de mise en œuvre [30]. Dans le cas de l'extrusion, la durée de séjour du polymère à l'état fondu est relativement longue (typiquement entre 1 et $2 \mathrm{~min})$. Il est maintenant bien établi que la thermo-oxydation du polymère est la principale source de problèmes $[24,31-$ 38]. Dans le cas de l'injection, bien que la durée de séjour du polymère à l'état fondu soit nettement plus brève (de l'ordre de quelques secondes), il semblerait que la thermo-oxydation joue aussi un rôle important [39-41]. Aux températures de mise en œuvre (typiquement entre 210 et $280{ }^{\circ} \mathrm{C}$ ), la thermo-oxydation est un mécanisme radicalaire en chaîne, essentiellement amorcé par la décomposition du principal produit de propagation : le groupe hydroperoxyde POOH [3, 4, 6, 9, 21, 24, 26, 27, 35, 36].

D'une manière générale, le vieillissement thermique du PET à l'état fondu conduit à deux grands types de modifications structurales :

- des modifications des groupes latéraux, c'est-à-dire principalement la formation de groupes hydroxyle et carbonyle $[5,6$, $9,11,12,15,16,21,23,24,26-28]$, mais aussi de diverses structures conjuguées $[3,5,6$, $8,9,15,16,20,23,26]$;

- des modifications de la structure macromoléculaire, c'est-à-dire des coupures [3$27,31-43]$ et des soudures de chaînes [3, $5,6,8-10,14,16,20,22-24,26,35,36]$.

Les modifications macromoléculaires affectent aussi bien le comportement rhéologique du polymère en solution ou à l'état fondu (variations de viscosité) [3,5$7,9,18,24,31-38,40-42]$, que le comportement mécanique du polymère à l'état solide (chute catastrophique des propriétés à la rupture) [27, 31, 33, 34, 38-41, 44], et ceci même aux très faibles conversions. Il est maintenant bien établi que les coupures de chaînes conduisent à la formation de nouvelles extrémités de chaînes aldéhyde $[5,6,9$, $16,18,21,43]$, formate [23,27], acide carboxylique [3-12, 14, 16, 18-24, 26, 27,33, 34, 39-42] et vinylester [3-10,12,14-16,18,20-24,26]. Si elles se produisent près d'une extrémité de chaîne, elles conduisent aussi à la formation 
de nombreux composés volatils de faibles masses molaires. Il s'agit par exemple, du monoxyde de carbone $[3,5,6,10,16,18,27]$, du dioxyde de carbone $[3,5,6,10,11,15,16,18$, $20,21,23,25-27]$, du méthane $[3,5,6,10]$, de l'éthylène $[3,5,6,10]$, du benzène $[3,5,6,27]$, mais aussi d'aldéhydes tels que le benzaldéhyde [10,18], le formaldéhyde [13, 15, 17, $21,27,43]$ et l'acétaldéhyde [3-18,20,22, 24 $26,43]$. L'acétaldéhyde serait, de loin, le produit majoritaire, puisqu'il représenterait environ $80 \%$ en moles de l'ensemble des composés volatils $[3,5,6]$. La formation d'aldéhydes pose un réel problème dans le secteur de l'emballage alimentaire, car ces molécules peuvent rester momentanément piégées dans la phase amorphe vitreuse du polymère semi-cristallin à température ambiante, et affecter, ensuite, les propriétés organoleptiques d'aliments ou de boissons sans saveur comme l'eau minérale.

Par contre, les soudures de chaînes (allongements de chaînes, branchements et, à terme, réticulation) conduisent à la formation de nouveaux groupes dans le squelette carboné de la chaîne. Malheureusement, la faible sensibilité des techniques d'analyse physico-chimiques courantes de laboratoire (spectrophotométrie IR, RMN solide $\mathrm{du}{ }^{13} \mathrm{C}$, etc.) ne permet pas de caractériser ces structures avec certitude. Il s'agirait essentiellement de groupes anhydride $[3,5,6,8-10,14,22,24,26,36]$, de groupes méthyne $[3,6,8,9,23,35,36,45]$, ou de structures bi- et tri-phényle [20,26].

En revanche, les modifications de groupes latéraux n'affectent pas le comportement rhéologique ou mécanique du polymère aux faibles conversions, mais plutôt d'autres propriétés plus spécifiques à son application, comme les propriétés électriques ou optiques. Le problème le plus important, en pratique, est la décoloration qui résulte de la présence de groupes chromophores, même parfois en très faible concentration. Les principaux groupes chromophores seraient des groupes anhydride $[3,5,6,8-12,14,16,22,24,26,27,36]$, des groupes phénol $[15,16,23,24,26,28]$ qui se transformeraient en cétones aromatiques de type quinone $[15,16,23,28]$, des cétoesters conjugués [26], diverses structures aromatiques conjuguées $[15,16,20,26]$, ou encore, des séquences polydiène $[3,5,6,8,9,23]$.

Le présent article est dédié à l'étude des modifications irréversibles de la structure macromoléculaire du PET pendant son recyclage mécanique par extrusion. L'objectif visé est double. Il s'agit, tout d'abord, de proposer une méthode suffisamment sensible pour mettre en évidence les conséquences de ces modifications sur le plan rhéologique. Il s'agit, ensuite, d'élaborer un modèle cinétique de vieillissement thermique du PET à haute température, à l'état fondu, pour identifier, parmi l'éventail de mécanismes réactionnels proposés dans la littérature, ceux qui seraient probablement responsables de ces modifications.

\section{Théorie}

\subsection{Premier constat}

Il existe donc un large consensus sur le fait que la thermo-oxydation est la principale source de problèmes pendant le recyclage mécanique du PET par extrusion [24,31-38]. Cependant, les avis divergent au sujet de l'évolution de l'architecture macromoléculaire (voir Tab. 1).

Les valeurs de la masse moléculaire moyenne en poids $M_{W}$ ont été portées en fonction du nombre d'opérations d'extrusion sur la figure 1. Ces valeurs ont été déterminées par viscosimétrie, soit en solution à température ambiante $[24,33,34]$, soit à l'état fondu à 270 ou $280^{\circ} \mathrm{C}[31,35,36]$, à l'aide de la loi de Mark-Houwink:

$$
\eta \approx K M_{W}^{\alpha}
$$

où $K$ est une fonction décroissante de la température et $\alpha \approx 0,7$ en solution (dans ce cas, $\eta=\mathrm{VI}$ et est appelée "viscosité intrinsèque ") et $\approx 3,4$ à l'état fondu (dans ce cas, $\eta$ est appelée « viscosité Newtonienne »).

Dans la grande majorité des cas de figure [24, 31-34, 36-38], le recyclage mécanique entraîne une large prédominance des coupures de chaînes, ce qui se traduit par une chute monotone de $M_{W}$ et de la viscosité. En revanche, dans deux cas de figure $[35,36]$, il entraîne aussi des soudures qui peuvent devenir, à terme, prédominantes sur les coupures de chaînes, ce qui se traduit alors par une ré-augmentation de $M_{W}$ et de la viscosité. Dans l'un d'entre eux (Assadi et al. [35]), on assiste même à une gélification (réticulation) du polymère dès la quatrième opération d'extrusion, ce qui entraîne un blocage complet de l'extrudeuse.

Une manière originale pour détecter la formation de structures branchées, en deçà du point de gel, a été proposée par Assadi et al. [35]. Elle consiste à comparer les valeurs de $M_{W}$ déterminées, à l'aide de deux 
Tableau 1. Modifications irréversibles de la structure macromoléculaire pendant le recyclage mécanique du PET par extrusion [24,31-37]. Les dimensions des extrudeuses et les conditions opératoires sont également rapportées.

Table 1. Irreversible macromolecular changes during PET mechanical recycling by extrusion [24,31-37]. The extruder geometry and size, and processing conditions are also reported.

\begin{tabular}{|c|c|c|c|c|c|c|}
\hline Références & $\begin{array}{l}\text { De Paoli } \\
{[24,34]}\end{array}$ & $\begin{array}{c}\text { La Mantia } \\
{[31,32]}\end{array}$ & $\begin{array}{c}\text { Frounchi } \\
\text { [33] }\end{array}$ & $\begin{array}{c}\text { Assadi } \\
\text { [35] }\end{array}$ & $\begin{array}{c}\text { Nait Ali } \\
{[36]}\end{array}$ & $\begin{array}{c}\text { Ribes-Greus } \\
\text { [37] }\end{array}$ \\
\hline Type & Wortex WEX & Brabender PLE & Collin $\mathrm{Nr}$ & Samafor BG & Fairex Compact & Brabender PL \\
\hline d'extrudeuse & 30 monovis & 640 monovis & 5500 bivis & 45 monovis & monovis & 2000 bivis \\
\hline Section d'alimentation $\left(\mathrm{mm}^{2}\right)$ & - & - & - & 1800 & 3800 & - \\
\hline Longueur de vis (cm) & 96 & 47,5 & - & 110 & 88 & 75 \\
\hline Section de tête $\left(\mathrm{mm}^{2}\right)$ & - & - & - & 63 & 80 & - \\
\hline Profil de température $\left({ }^{\circ} \mathrm{C}\right)$ & $220-280$ & $210-270$ & $235-260$ & $240-280$ & $220-260$ & $250-270$ \\
\hline Vitesse (rpm) & 102 & 100 & 62 & 40 & 80 & - \\
\hline Temps de résidence (s) & 120 & - & - & 90 & 50 & - \\
\hline $\begin{array}{l}\text { Principaux mécanismes } \\
\text { de dégradation }\end{array}$ & $\begin{array}{l}\text { Coupures } \\
\text { de chaînes }\end{array}$ & $\begin{array}{l}\text { Coupures } \\
\text { de chaînes }\end{array}$ & $\begin{array}{l}\text { Coupures } \\
\text { chaînes }\end{array}$ & $\begin{array}{c}\text { Coupures et } \\
\text { soudures de } \\
\text { chaînes }\end{array}$ & $\begin{array}{l}\text { Coupures et } \\
\text { soudures de } \\
\text { chaînes }\end{array}$ & $\begin{array}{l}\text { Coupures } \\
\text { de chaînes }\end{array}$ \\
\hline
\end{tabular}

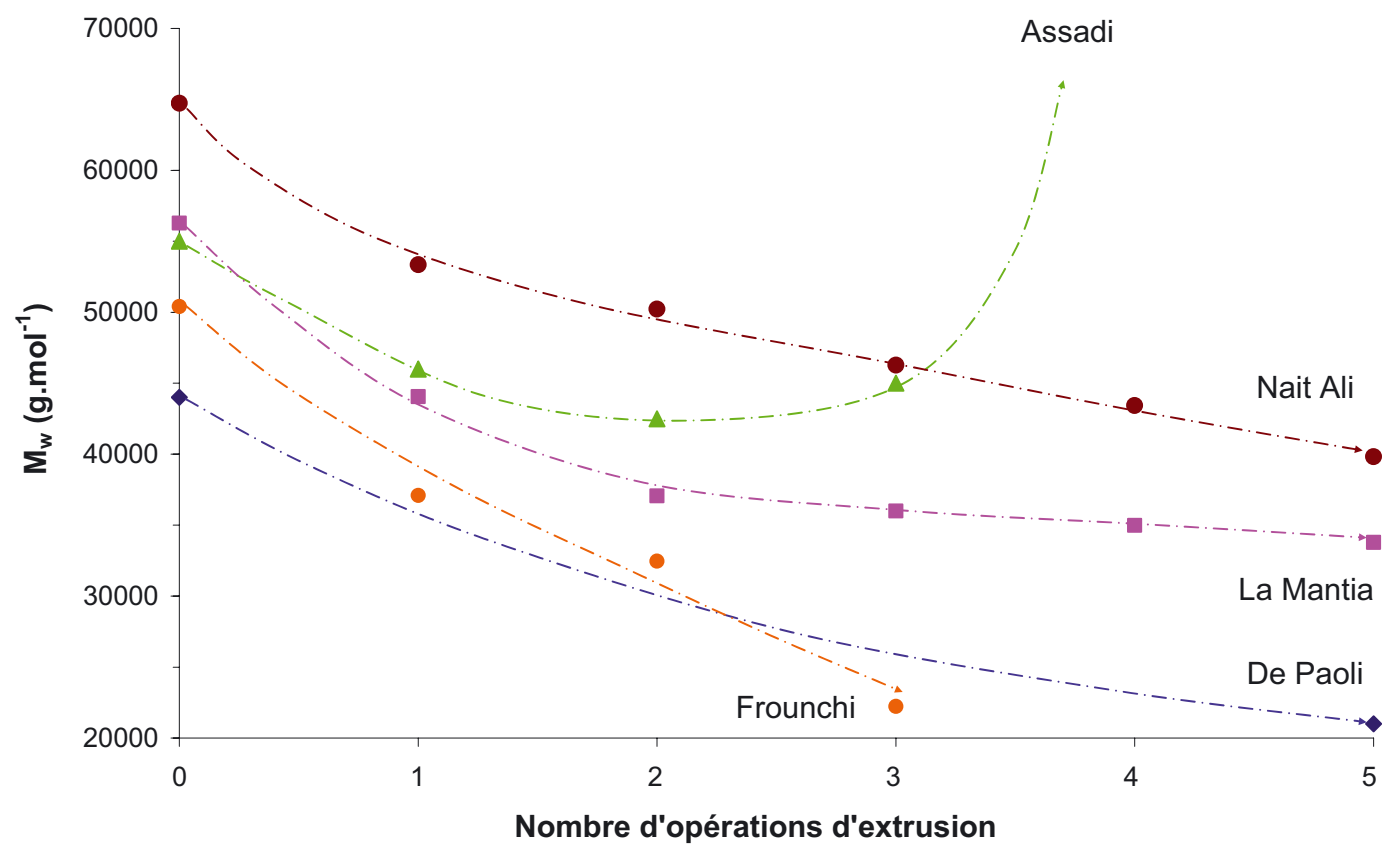

Fig. 1. Variations de la masse molaire moyenne en poids en fonction du nombre d'opérations d'extrusion d'après différents auteurs [24,31,33-36].

Fig. 1. Changes in weight average molar mass versus number of extrusion cycles according to different authors $[24,31,33-36]$.

techniques analytiques différentes : la viscosimétrie (à l'état fondu) et la chromatographie d'exclusion stérique (voir Fig. 2). Si le polymère restait linéaire (cas du polymère initial), c'est-à-dire s'il n'y avait que des coupures et des allongements de chaînes, les valeurs de $M_{W}$ seraient identiques. Cependant, comme des branchements de chaînes se produisent simultanément, les valeurs sont différentes dès les premières opérations d'extrusion. Ce résultat n'est pas surprenant car, pour une masse molaire identique, la présence de branches gêne la reptation de la chaîne dans le fondu, mais réduit aussi le volume hydrodynamique de la chaîne en solution. Au final, on observe deux évolutions totalement différentes de $M_{W}$ en fonction du nombre d'opérations d'extrusion : la courbe viscosimétrique présente un minimum dès la deuxième opération d'extrusion, attribué à un équilibre entre coupures et branchements de chaînes, alors que la courbe chromatographique présente une allure sigmoïdale.

Une manière plus classique pour détecter la présence de structures branchées consiste à comparer les valeurs de VI de PET recyclés et d'une série de PET linéaires, pris comme matériaux de référence (voir Fig. 3) [35]. En effet, ces deux familles de polymères obéissent à la loi précédente de Mark-Houwink, mais pour des valeurs 


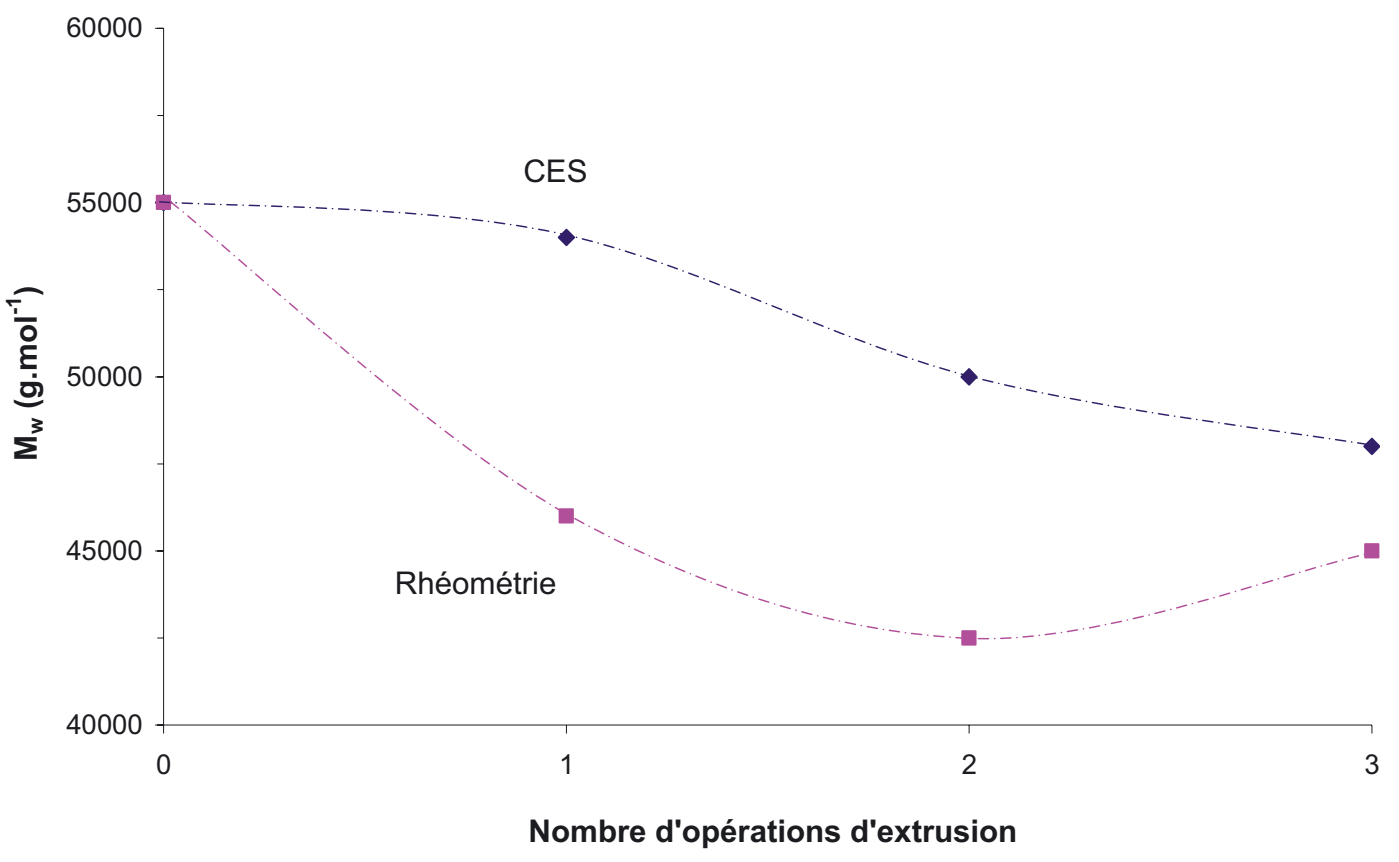

Fig. 2. Variations de la masse moléculaire moyenne en poids en fonction du nombre d'opérations d'extrusion d'après Assadi et al. [35]. Comparaison des masses déterminées par viscosimétrie (à l'état fondu) et par chromatographie d'exclusion stérique (CES).

Fig. 2. Changes in weight average molar mass versus number of extrusion cycles according to Assadi et al. [35]. Comparison between average molar masses determined by viscosimetry (in molten state) and by steric exclusion chromatography (SEC).

d'exposant $\alpha$ relativement différentes $(\approx 0,97$ pour les recyclés contre $\approx 0,77$ pour les linéaires). De plus, on note que les points des PET recyclés se situent clairement en dessous de la droite caractéristique des PET linéaires, et leur distance à cette droite augmente avec le nombre d'opérations d'extrusion. Ainsi, pour les conditions opératoires d'extrusion étudiées par Assadi et al. [35], il semblerait que le nombre des branchements augmente de manière monotone avec le nombre d'opérations d'extrusion. Cependant, cette évolution du comportement rhéologique semble être, en première approche, très éloignée de celle rapportée par les autres auteurs dans la littérature [24,31-34,36-38]. Comment expliquer une telle différence de comportements?

Une piste de recherche, qui nous a parue intéressante, est la suivante : si l'on parcourt attentivement le tableau 1, malgré un manque fragrant d'informations sur les conditions opératoires d'extrusion dans certains cas de figure, il semblerait que les coupures de chaînes soient largement favorisées dans le cas d'une extrudeuse «bien aérée » (sections d'alimentation et de tête larges, et vis courte), et que les soudures de chaînes ne soient détectées que dans le cas d'extrudeuses "peu aérées » (sections d'alimentation et de tête étroites, et vis longue). Nous en déduisons que la concentration d'oxygène devait être un paramètre clé de la dégradation thermique du PET à l'état fondu. Nous avons ainsi cherché à vérifier cette hypothèse.

\subsection{Proposition de schéma simplifié d'extrudeuse}

Bien que, en conditions réelles d'extrusion, le problème à résoudre soit bien plus compliqué - notamment en raison du transport mécanique de particules de polymère oxydées le long de la vis - nous avons choisi, en première approche, de représenter le réacteur d'une extrudeuse sous une forme relativement simple. Nous sommes partis du constat que le polymère fondu est confiné dans la zone pressurisée et ne se trouve en contact avec l'air, à pression atmosphérique, qu'au niveau des deux extrémités de l'extrudeuse (l'alimentation et la tête). C'est donc au niveau de ces deux extrémités que la concentration d'oxygène est la plus élevée. On s'attend à ce que cette dernière diminue rapidement vers le centre du réacteur, puisque la diffusion d'oxygène est, en général, nettement plus lente que sa réaction 


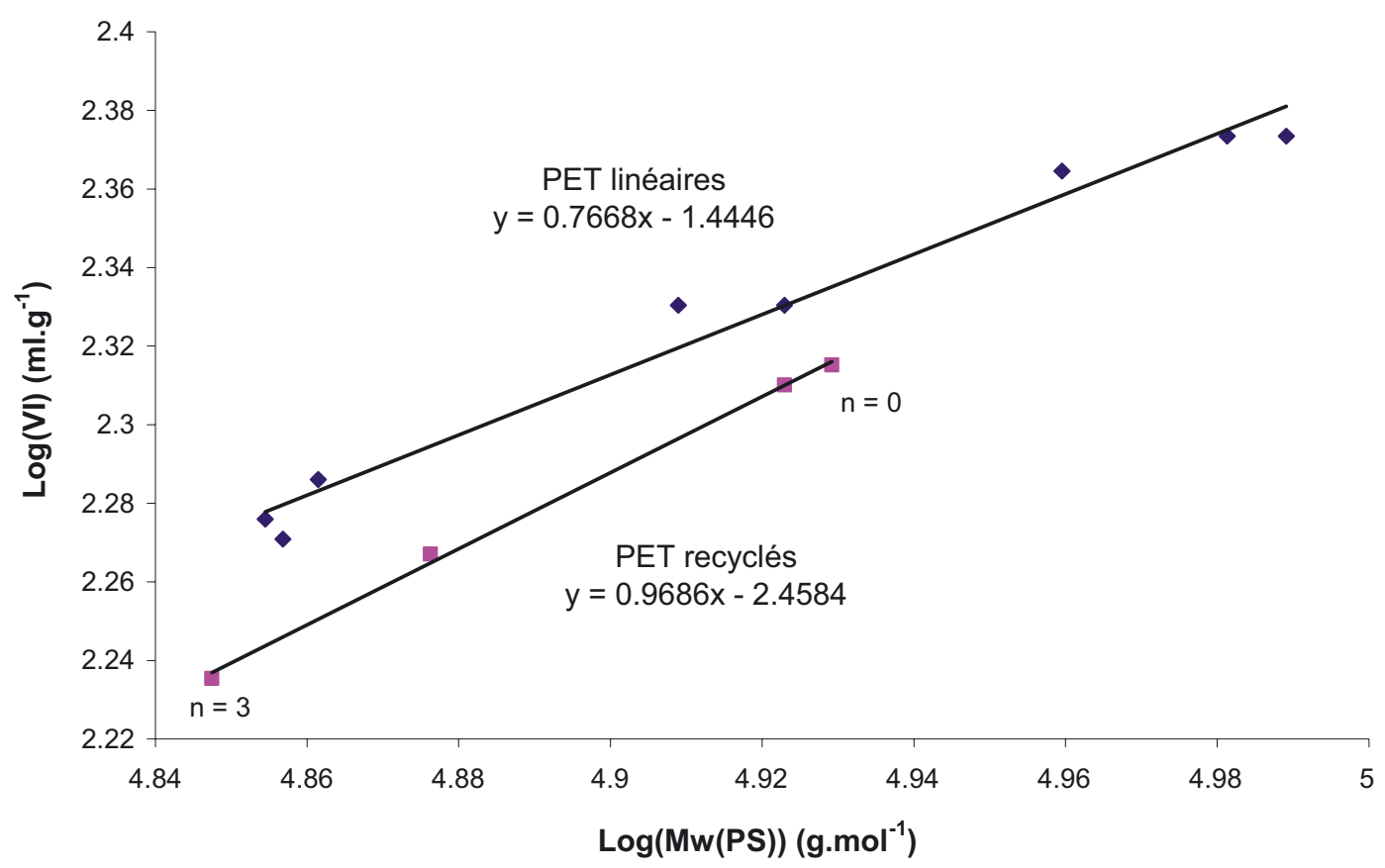

Fig. 3. Variations de la viscosité intrinsèque en fonction de la masse moléculaire moyenne en poids déterminée par CES. Comparaison des viscosités des PET recyclés et des PET linéaires de référence [35].

Fig. 3. Changes in intrinsic viscosity versus weight average molar mass determined by SEC. Comparison between recycled and linear (taken as a reference) PET viscosities [35].

avec le polymère [46]. Cependant, comme la température n'est pas homogène dans le réacteur, la thermo-oxydation du polymère fondu va se produire en régime non isotherme.

Les gradients de concentration d'oxygène et de température ont été schématisés sur la figure 4 . Les coordonnées $z_{0}$ et $z_{L}$ indiquent les positions des interfaces air/polymère fondu au niveau des zones d'alimentation et de tête, respectivement. Ainsi, la longueur de la vis s'écrit :

$$
L=z_{L}-z_{0} .
$$

Les coordonnées $z_{1}$ et $z_{2}$ délimitent les deux zones "fortement oxygénées » (1 et 3), dans lesquelles les coupures de chaînes devraient largement prédominer, de la zone "sous-oxygénée » (2) dans laquelle les soudures de chaînes devraient être favorisées. Ainsi, les longueurs de ces différentes zones s'écrivent :

$$
\begin{aligned}
& L_{1}=z_{1}-z_{0} \\
& L_{2}=z_{2}-z_{1} \\
& L_{3}=z_{L}-z_{2} .
\end{aligned}
$$

La concentration d'équilibre d'oxygène, aux interfaces air/polymère fondu, obéit à la loi de Henry :

$$
\left[\mathrm{O}_{2}\right]\left(z_{i}\right)=S_{\text {fondu }}\left(T_{i}\right) \times P_{\mathrm{O}_{2}} .
$$

On sait que la pression partielle d'oxygène $P_{\mathrm{O}_{2}}$ dans l'air ambiant est de l'ordre de $21 \%$ de la pression atmosphérique, c'est-à-dire $2,1 \times 10^{4} \mathrm{~Pa}$. De plus, en première approche, on peut estimer la solubilité d'oxygène dans le fondu à partir de la solubilité mesurée dans le polymère semi-cristallin au dessus de sa température de transition vitreuse, connaissant son taux de cristallinité :

$$
S_{\text {fondu }}=\frac{S_{\text {semi-crist }}}{1-\chi_{\mathrm{C}}} \text {. }
$$

Les bases de données [par ex. 47,48] montrent que la solubilité d'oxygène dans les polymères est pratiquement indépendante de la température. L'application numérique des équations (4) et (5), en prenant $S_{\text {semi-crist }}=3,1 \times 10^{-8} \mathrm{moll}^{-1} \mathrm{~Pa}^{-1}$ [48] et $\chi_{\mathrm{C}}=33 \%$, conduit finalement à :

$$
S_{\text {fondu }}=4,6 \times 10^{-8} \mathrm{moll}^{-1} \mathrm{~Pa}^{-1}
$$

i.e.

$\left[\mathrm{O}_{2}\right]\left(z_{0}\right)=\left[\mathrm{O}_{2}\right]\left(z_{1}\right)=\left[\mathrm{O}_{2}\right]_{\mathrm{S}}=9,7 \times 10^{-4} \mathrm{moll}^{-1}$.

Le seul paramètre inconnu est donc la concentration critique d'oxygène, aux interfaces zones «fortement oxygénées »/zone « sous-oxygénée » :

$$
\left[\mathrm{O}_{2}\right]\left(z_{1}\right)=\left[\mathrm{O}_{2}\right]\left(z_{2}\right)=\left[\mathrm{O}_{2}\right]_{C} .
$$




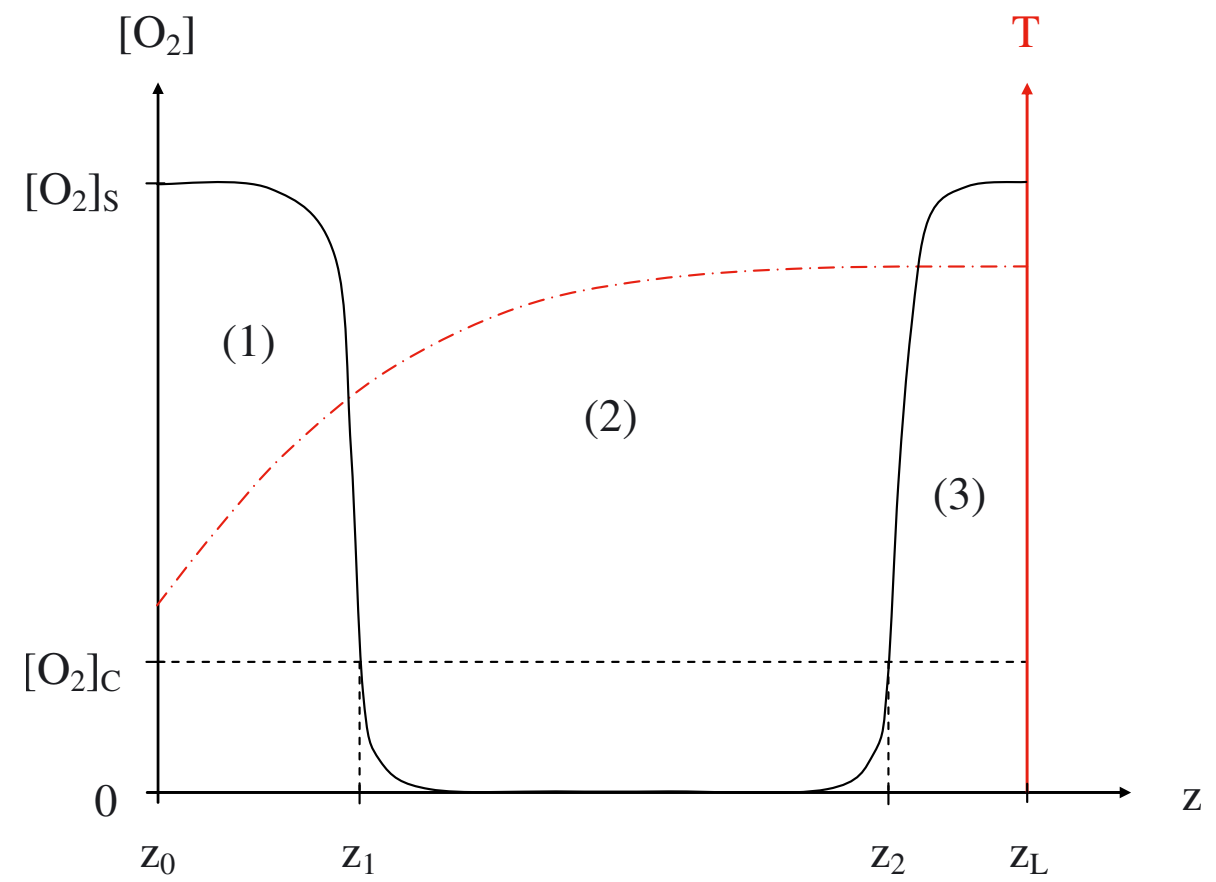

Fig. 4. Schéma simplifié d'une extrudeuse. $\left[\mathrm{O}_{2}\right]$ et $T$ sont les gradients respectifs d'oxygène et de température dans le réacteur. Les positions $z_{i}$ délimitent les différentes zones "réactionnelles » (1, 2 et 3$)$ dans le réacteur (voir texte).

Fig. 4. Simplified scheme of an extruder reactor. $\left[\mathrm{O}_{2}\right]$ and $\mathrm{T}$ designate respectively the oxygen concentration and temperature gradients in the reactor. Positions $z_{i}$ are the limits between the different "reaction" zones $(1,2$ and 3) in the reactor (see text).

Cette dernière sera déterminée, dans cette étude, à partir d'essais de thermo-oxydation ciblés sous différentes pressions partielles d'oxygène.

D'après ce schéma simplifié, les coupures et les soudures de chaînes vont se produire successivement pendant une opération d'extrusion, puisque chacune d'entre elles est largement favorisée dans une zone distincte $d u$ réacteur : les zones «fortement oxygénées » et la zone «sous-oxygénée», respectivement. Ainsi, au bilan, la prédominance relative de ces deux types de modifications macromoléculaires dépend de 4 facteurs :

- la longueur de la vis $L$;

- les longueurs des deux zones «fortement oxygénées » $L_{1}$ et $L_{3}$;

- et le temps de résidence $t_{R}$.

Les longueurs $L_{1}$ et $L_{3}$ dépendent, à leur tour, de 5 variables :

- la concentration d'équilibre $\left[\mathrm{O}_{2}\right]_{S}$ et la vitesse de consommation d'oxygène $v\left(\left[\mathrm{O}_{2}\right]_{\mathrm{S}}\right)$ aux interfaces air/polymère fondu ;

- la diffusivité d'oxygène $D_{\mathrm{O}_{2}}$ dans le fondu ;

- le profil de température dans le réacteur;

- et la vitesse de transport du polymère le long de la vis.
Nous avons vu que $\left[\mathrm{O}_{2}\right]_{S}$ est indépendante de la température (voir Éq. (4)). En revanche, le rapport $D_{\mathrm{O}_{2}} / v\left(\left[\mathrm{O}_{2}\right]_{S}\right)$ est une fonction décroissante de la température [46]. On s'attend donc à ce que : $L_{1}>L_{3}$.

À ce stade, on peut définir deux cas limites d'extrudeuses :

- les extrudeuses «bien aérées", pour lesquelles $L_{1}>L_{3} \gg L_{2}$, conduisant à des coupures de chaînes ;

- et les extrudeuses "peu aérées", pour lesquelles $L_{2} \gg L_{1}>L_{3}$, conduisant plutôt à des soudures de chaînes.

Ce schéma simplifié devrait donc suffire à rendre compte de l'ensemble des évolutions des comportements rhéologiques observées dans la littérature pendant le recyclage mécanique du PET.

\subsection{Utilisation du rhéomètre comme outil de simulation des modifications macromoléculaires}

Une solution originale, pour tenter de valider ce schéma simplifié, consiste à étudier les modifications macromoléculaires induites par thermo-oxydation du PET à l'état fondu dans des conditions d'exposition (en 
température, oxygénation et cisaillement) soigneusement contrôlées. La cavité d'un rhéomètre convient parfaitement pour ce genre d'étude, pour les raisons suivantes :

- le champ de température est homogène et parfaitement régulé ;

- l'atmosphère peut être parfaitement contrôlée et rapidement changée ;

- les amplitudes de cisaillement sont suffisamment faibles pour garantir l'absence de processus mécano-chimique ;

- l'appareil donne une information directe de la masse moléculaire moyenne en poids $M_{W}$ avec une très grande sensibilité.

Cet appareil peut donc être utilisé pour réaliser deux essais de vieillissement thermique :

- des essais destinés à reproduire les modifications macromoléculaires locales (prédominance de coupures ou de soudures de chaînes) à une concentration d'oxygène donnée, c'est-à-dire à une position $z$ dans le réacteur d'extrusion. Il s'agit d'essais isothermes à une pression partielle d'oxygène constante, comprise entre $0 \%$ (azote) et 21 \% (air ambiant) de la pression atmosphérique ;

- des essais destinés à reproduire l'historique des modifications macromoléculaires (successions de coupures et soudures de chaînes) pendant une opération complète d'extrusion. Il s'agit d'essais isothermes à une pression partielle d'oxygène variable entre $0 \%$ et $21 \%$ de la pression atmosphérique, pendant lesquels le polymère est exposé sous azote, mais avec de brèves admissions (pendant 2 min) d'air ambiant.

\subsection{Proposition de modèle cinétique}

Le point de départ est le schéma d'oxydation en «boucle fermée » établi pour le polyéthylène $(\mathrm{PE})$, à température basse à modérée (entre 40 et $220^{\circ} \mathrm{C}$ ), dans une étude antérieure [49]. En effet, on s'attend à ce que le PET se comporte essentiellement comme un substrat poly(méthylénique) vis-à-vis de l'oxydation. Cependant, dans le domaine de températures explorées $\left(T \geq 250{ }^{\circ} \mathrm{C}\right)$, on peut envisager des modifications de ce schéma :

- la concentration critique en hydroperoxydes $[\mathrm{POOH}]_{\mathrm{C}}$, au-delà de laquelle la décomposition thermique des $\mathrm{POOH}$ passe du mode unimoléculaire au mode bimoléculaire, est une fonction croissante de la température. Elle est de l'ordre de $9,2 \times 10^{-1} \mathrm{moll}^{-1}$ à $250{ }^{\circ} \mathrm{C}$, c'est-à-dire qu'il faudrait accumuler une quantité colossale de $\mathrm{POOH}$ pour enclencher le mode bimoléculaire. Ainsi, au dessus de $250{ }^{\circ} \mathrm{C}$, on peut raisonnablement considérer que la décomposition thermique des $\mathrm{POOH}$ est essentiellement unimoléculaire;

- l'oxygène moléculaire étant un bi-radical à l'état fondamental, on ne peut pas exclure sa réaction directe avec le polymère :

\begin{tabular}{ll}
$\mathrm{PH}+\mathrm{O}_{2}$ & $\rightarrow \mathrm{P}^{\circ}+\mathrm{HO}_{2}^{\circ} \quad\left(k_{10}\right)$ \\
$\mathrm{HO}_{2}^{\circ}+\mathrm{PH}$ & $\rightarrow \mathrm{P}^{\circ}+\mathrm{H}_{2} \mathrm{O}_{2}$ \\
$\mathrm{H}_{2} \mathrm{O}_{2}$ & $\rightarrow 2 \mathrm{HO}^{\circ}$ \\
$2 \mathrm{HO}^{\circ}+2 \mathrm{PH}$ & $\rightarrow 2 \mathrm{P}^{\circ}+2 \mathrm{H}_{2} \mathrm{O}$ \\
\hline (1o) Réaction $\mathrm{PH}+\mathrm{O}_{2}$ & $\rightarrow 4 \mathrm{P}^{\circ}+2 \mathrm{H}_{2} \mathrm{O}\left(k_{10}\right) ;$ \\
bilan &
\end{tabular}

- au dessus de $250{ }^{\circ} \mathrm{C}$, les ponts peroxydes POOP ne peuvent pas survivre. La dismutation est donc la seule terminaison possible des paires de radicaux alkoxy. Elle conduit à la formation de groupes anhydride et alcool :
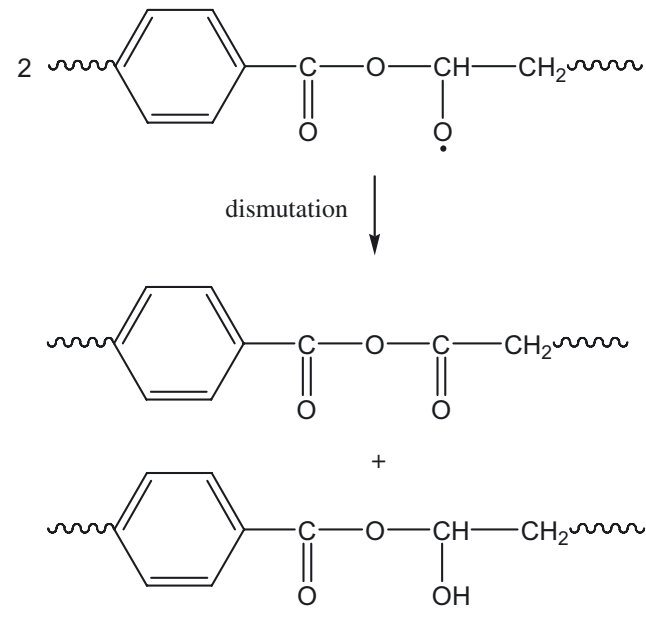

- les aldéhydes sont les principaux produits des coupures de chaînes. Ils sont formés par coupure $\beta$ des radicaux alkoxy. Cependant, leur liaison C-H présente une énergie de dissociation nettement plus faible $\left(E_{D} \approx 368 \mathrm{~kJ} \mathrm{~mol}^{-1}\right)$ que celle des méthylènes $\left(E_{D} \approx 393 \mathrm{~kJ} \mathrm{~mol}^{-1}\right)$. On s'attend donc à ce qu'ils s'oxydent rapidement en acides carboxyliques (voir schéma 1). 

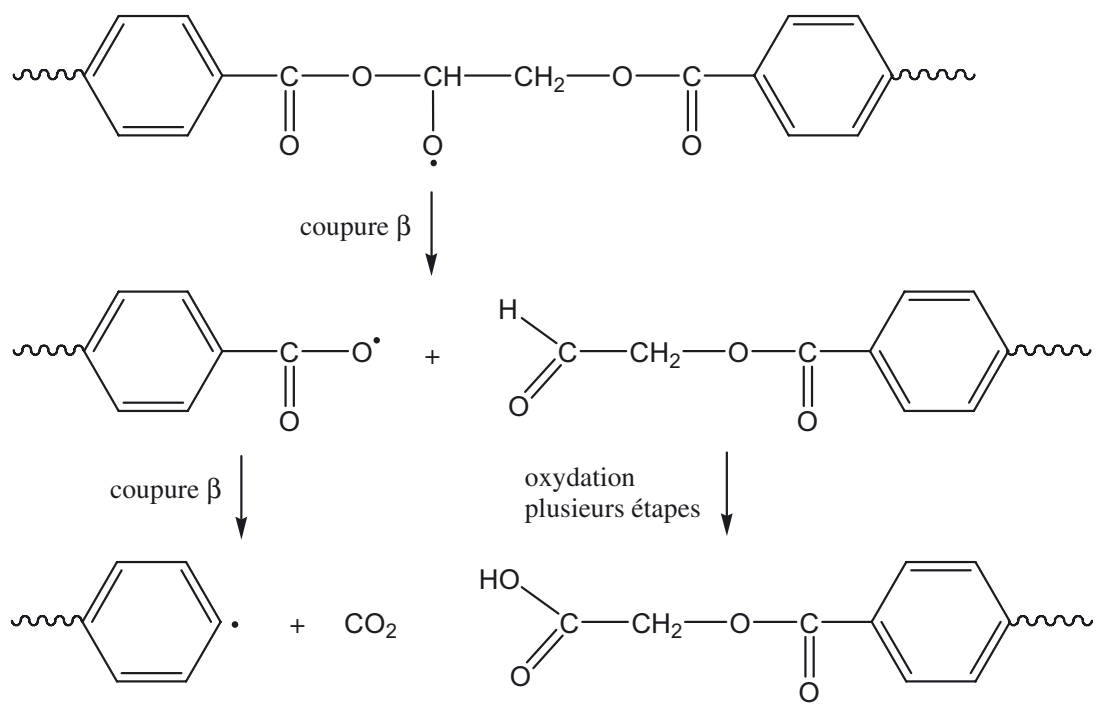

Schéma 1.

Cette hypothèse se justifie pleinement car, en règle générale, lorsqu'ils sont détectés, les aldéhydes représentent une infime proportion des produits d'oxydation formés pendant le recyclage mécanique du PET par extrusion. On peut donc, en première approche, remplacer tous les aldéhydes par des acides carboxyliques dans le schéma mécanistique d'oxydation.

Au final, le schéma mécanistique d'oxydation du PET à l'état fondu pourrait s'écrire (voir schéma 2), où $\mathrm{PH}$ désigne une liaison $\mathrm{C}-\mathrm{H}$ d'un méthylène, $\mathrm{POOH}$ un hydroperoxyde, $\mathrm{P}^{\circ}$ un radical alkyl, $\mathrm{PO}_{2}^{\circ}$ un radical peroxy, $\left[\mathrm{PO}^{\circ}{ }^{\circ} \mathrm{OP}\right]_{\text {cage }}$ une paire de radicaux alkoxy dans une cage, $\mathrm{POH}=\mathrm{O}$ un acide, $\mathrm{O}(\mathrm{P}=\mathrm{O})_{2}$ un anhydride, $\mathrm{POH}$ un alcool, $\mathrm{PP}$ un pont carbone-carbone, $\mathrm{F}$ une double liaison vinyle, $S$ une coupure de chaîne et $X$ un branchement de chaînes. Les coefficients $\gamma_{1}$ et $\gamma_{4}$ sont les rendements respectifs en coupures et en branchements de chaînes dans les réactions concernées.

De plus, nous avons vu, en introduction, que plusieurs processus non radicalaires sont susceptibles de se superposer à l'oxydation. Parmi l'éventail de mécanismes proposés, deux font l'objet d'un relatif consensus dans la littérature et, pour cette raison, ont particulièrement retenu notre attention. Il s'agit, d'une part, de la décomposition thermique des groupes ester des principales irrégularités structurales, en l'occurrence les unités diéthylène glycol :

(0i) Décomposition Irreg $\rightarrow \mathrm{POH}=\mathrm{O}+\mathrm{F}_{V}$ $+\mathrm{S}\left(k_{0 i}\right)$

où $\mathrm{F}_{v}$ désigne une double liaison vinylidène et $+\mathrm{S}$ une coupure de chaîne.

Il s'agit, d'autre part, de la condensation des principaux produits d'amorçage (voir réactions $1 \mathrm{u}$ et 63), en l'occurrence des acides carboxyliques :

(7) Condensation $\mathrm{POH}=\mathrm{O}+\mathrm{POH}=\mathrm{O} \rightarrow$ $\mathrm{O}(\mathrm{P}=\mathrm{O})_{2}-\mathrm{S} \quad\left(k_{7}\right)$

où $-\mathrm{S}$ désigne un allongement de chaines.

À ce stade, on remarquera qu'un acte de condensation conduit à un allongement et non à un branchement de chaîne. Il s'agit donc d'une réaction inverse à une coupure de chaîne.

Nous avons donc retenu le schéma mécanistique, constitué des 11 réactions

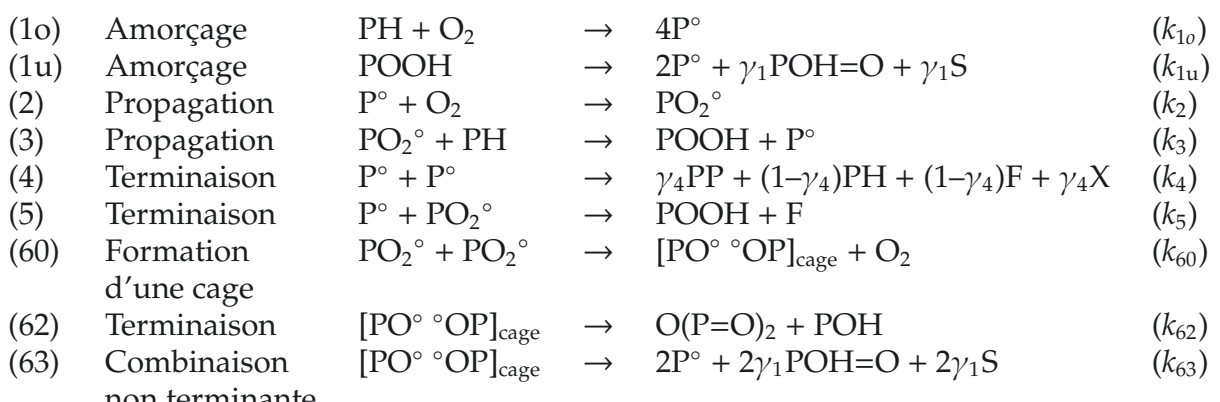


élémentaires précédentes $\{0 \mathrm{i}, 1 \mathrm{u}, 1 \mathrm{o}, 2,34$, $5,60,62,63$ et 7$\}$, pour tenter de décrire la cinétique de vieillissement thermique du PET à l'état fondu dans cette étude. Dans le cas simplifié d'une oxydation homogène (i.e. en absence de gradients d'oxydation), la concentration d'oxygène dans le polymère reste constante en tout point du polymère. L'équation (4) peut donc être réécrite sous la forme suivante :

$$
\left[\mathrm{O}_{2}\right]=4,6 \times 10^{-8} \times \mathrm{P}_{\mathrm{O}_{2}} .
$$

Ainsi, le schéma mécanistique conduit à un système de 7 équations différentielles :

$$
\begin{aligned}
\frac{\mathrm{d}[\text { Irreg }]}{\mathrm{d} t}= & -k_{0 i}[\text { Irreg }] \\
\frac{\mathrm{d}[\mathrm{POOH}]}{\mathrm{d} t}= & -k_{1 u}[\mathrm{POOH}]+k_{3}[\mathrm{PH}]\left[\mathrm{PO}_{2}^{\bullet}\right] \\
& +k_{5}\left[\mathrm{P}^{\bullet}\right]\left[\mathrm{PO}_{2}^{\bullet}\right] \\
\frac{\mathrm{d}\left[\mathrm{P}^{\bullet}\right]}{\mathrm{d} t}= & 4 k_{1 o}[\mathrm{PH}]\left[\mathrm{O}_{2}\right]+2 k_{1 u}[\mathrm{POOH}] \\
& -k_{2}\left[\mathrm{O}_{2}\right]\left[\mathrm{P}^{\bullet}\right]+k_{3}[\mathrm{PH}]\left[\mathrm{PO}_{2}^{\bullet}\right] \\
& -2 k_{4}\left[\mathrm{P}^{\bullet}\right]^{2}-k_{5}\left[\mathrm{P}^{\bullet}\right]\left[\mathrm{PO}_{2}^{\bullet}\right] \\
& +2 k_{63}\left[\mathrm{PO}^{\bullet \bullet} \mathrm{OP}\right]_{\mathrm{cage}} \\
\frac{\mathrm{d}\left[\mathrm{PO}_{2}^{\bullet}\right]}{\mathrm{d} t}= & k_{2}\left[\mathrm{O}_{2}\right]\left[\mathrm{P}^{\bullet}\right]-k_{3}\left[\mathrm{PH}_{[}\right]\left[\mathrm{PO}_{2}^{\bullet}\right] \\
& -k_{5}\left[\mathrm{P}^{\bullet}\right]\left[\mathrm{PO}_{2}^{\bullet}\right]-2 k_{60}\left[\mathrm{PO}_{2}^{\bullet}\right]^{2}
\end{aligned}
$$

$$
\begin{aligned}
\frac{\mathrm{d}\left[\mathrm{PO}^{\bullet \bullet} \mathrm{OP}\right]_{\text {cage }}}{\mathrm{d} t}= & 2 k_{60}\left[\mathrm{PO}_{2}^{\bullet}\right]^{2} \\
& -\left(k_{62}+k_{63}\right)\left[\mathrm{PO}^{\bullet \bullet} \mathrm{OP}\right]_{\text {cage }}
\end{aligned}
$$

$$
\begin{aligned}
& \frac{\mathrm{d}[\mathrm{PH}]}{\mathrm{d} t}=-2 k_{0 i}[\text { Irreg }]-4 k_{1 o}[\mathrm{PH}]\left[\mathrm{O}_{2}\right] \\
& -\left(2-\gamma_{1}\right) k_{1 u}[\mathrm{POOH}]-k_{3}[\mathrm{PH}]\left[\mathrm{PO}_{2}^{\circ}\right] \\
& -k_{5}\left[\mathrm{P}^{\circ}\right]\left[\mathrm{PO}_{2}^{\circ}\right]-2\left(1-\gamma_{1}\right) k_{63}\left[\mathrm{PO}^{\bullet \bullet} \mathrm{OP}\right]_{\text {cage }}
\end{aligned}
$$

$$
\begin{aligned}
& \frac{\mathrm{d}[\mathrm{POH}=\mathrm{O}]}{\mathrm{d} t}=k_{0 i}[\text { Irreg }]+\gamma_{1} k_{1 u}[\mathrm{POOH}] \\
& +2 \gamma_{1} k_{63}\left[\mathrm{PO}^{\bullet \bullet} \mathrm{OP}\right]_{\text {cage }}-2 k_{7}[\mathrm{POH}=\mathrm{O}]^{2}
\end{aligned}
$$

qui admet pour conditions initiales $(t=0)$ :

- $[$ Irreg $]=[\text { Irreg }]_{0}$;

$-[\mathrm{POOH}]=[\mathrm{POOH}]_{0}$;

- $\left[\mathrm{P}^{\bullet}\right]=\left[\mathrm{PO}_{2}^{\bullet}\right]=\left[\mathrm{PO}^{\bullet \bullet} \mathrm{OP}\right]_{\mathrm{cage}}=0$;

$-[\mathrm{PH}]=[\mathrm{PH}]_{0} \approx 14 \mathrm{moll}^{-1}$;

- Et $[\mathrm{POH}=\mathrm{O}]=[\mathrm{POH}=\mathrm{O}]_{0}=M_{n 0}^{-1}$.

(si l'on considère que les chaînes de PET sont initialement constituées d'une extrémité acide carboxylique et d'une extrémité hydroxyle éthylester).
Nous avons résolu numériquement le système d'équations différentielles (9) à (15) à l'aide du solveur ODE23s du logiciel commercial Matlab ${ }^{\circledR}$. Il s'agit d'un solveur basé sur l'algorithme semi-implicite de Rosenbrock, spécialement adapté pour la résolution des problèmes raides de cinétique chimique.

Ce système donne accès aux variations des concentrations des différentes espèces réactives : [Irreg], $[\mathrm{POOH}],\left[\mathrm{P}^{\bullet}\right],\left[\mathrm{PO}_{2}^{\bullet}\right]$, $\left[\mathrm{PO}{ }^{\bullet} \mathrm{OP}\right]_{\text {cage, }}[\mathrm{PH}]$ et $[\mathrm{POH}=\mathrm{O}]=\mathrm{f}(t)$. À partir de ces grandeurs, il est possible de calculer les vitesses des modifications macromoléculaires (i.e. des coupures et soudures de chaînes) :

$$
\begin{aligned}
\frac{\mathrm{dS}}{\mathrm{d} t}= & k_{0 i}[\text { Irreg }]+\gamma_{1} k_{1 u}[\mathrm{POOH}] \\
& +2 \gamma_{1} k_{63}[\mathrm{PO} \bullet \mathrm{OP}]_{\text {cage }}-k_{7}[\mathrm{POH}=\mathrm{O}]^{2} \\
\frac{\mathrm{dX}}{\mathrm{d} t} & =\gamma_{4} k_{4}\left[\mathrm{P}^{\circ}\right]^{2} .
\end{aligned}
$$

Les deux équations de Saito [50] permettent, ensuite, d'accéder aux variations des masses moléculaires moyennes en nombre $M_{n}$ et en poids $M_{W}$ :

$$
\begin{gathered}
S-X=\frac{1}{M_{n}}-\frac{1}{M_{n 0}} \\
\frac{S}{2}-2 X=\frac{1}{M_{W}}-\frac{1}{M_{W 0}}
\end{gathered}
$$

Enfin, les variations de viscosité Newtonienne peuvent être calculées à l'aide de la classique loi d'échelle [51,52] :

$$
\eta \approx K M_{W}^{3,4}
$$

avec $K=3,4 \times 10^{-4} \operatorname{Pas}\left(\mathrm{mol} \mathrm{kg}^{-1}\right)^{3,4}$ à $280{ }^{\circ} \mathrm{C}$ [34].

Le modèle cinétique constitué des équations (8) à (20) a été utilisé, dans cette étude, pour tenter de déterminer quelles sont les modifications macromoléculaires responsables des variations du comportement rhéologique du PET pendant son recyclage mécanique par extrusion.

\section{Matériaux et techniques}

\subsection{Matériaux}

L'étude a porté sur deux lots de PET régénérés fournis par la société SOREPLA (Neufchâteau, sud de la France). Dans les deux cas, les paillettes présentent un faible niveau de contaminants (quantité de PE $<30$ ppm, PVC 
Tableau 2. Principales caractéristiques des deux lots de PET régénérés étudiés.

Table 2. Main characteristics of both batches of regenerated PET under study.

\begin{tabular}{ccc}
\hline Références & Assadi [35] & Nait Ali [36] \\
\hline Lot de PET & PET 1 & PET 2 \\
$M_{W}\left(\mathrm{~kg} \mathrm{~mol}^{-1}\right)$ & 55 & 63,2 \\
$T_{F}\left({ }^{\circ} \mathrm{C}\right)$ & 250 & $240-250$ \\
$\mathrm{X}_{C}(\%)$ & 33 & 33 \\
$\rho$ & - & 1,41 \\
\hline
\end{tabular}

$<65$ ppm, métaux <5 ppm, PAN <10 ppm et papier $<5 \mathrm{ppm})$, ce qui les destine à des applications dans le secteur de l'emballage alimentaire. Elles se différencient essentiellement par leur masse moléculaire initiale $M_{W}$. Leurs principales caractéristiques sont rapportées dans le tableau 2.

\subsection{Essais de rhéométrie}

Les paillettes ont été soigneusement séchées pendant $15 \mathrm{~h}$ à $120{ }^{\circ} \mathrm{C}$ sous vide primaire pour réduire la teneur en humidité à des valeurs inférieures à 10 ppm et, ainsi, limiter les risques d'hydrolyse au cours de la mise en œuvre à haute température.

Les propriétés rhéologiques à l'état fondu des deux lots de PET ont ensuite été mesurées à $280{ }^{\circ} \mathrm{C}$ sous azote à l'aide d'un rhéomètre Rheometrics Ares et d'un dispositif à plateaux parallèles coaxiaux (diamètre $=50 \mathrm{~mm}$, entrefer $=1 \mathrm{~mm}$ ). Des essais à fréquence angulaire variable $(\omega=$ $10^{-2}-10^{2} \mathrm{rad} \mathrm{s}^{-1}$ ) ont été réalisés avec une amplitude de déformation $\gamma_{\max }$ de $20 \%$. Ces essais montrent que le comportement rhéologique des deux PET est Newtonien dans le domaine des faibles fréquences angulaires, typiquement pour $\omega \leq 10 \mathrm{rad} \mathrm{s}^{-1}$ (voir Fig. 5).

Ces conditions opératoires $\left(T=280{ }^{\circ} \mathrm{C}\right.$, $\gamma_{\max }=20 \%$ et $\omega=10 \mathrm{rad} \mathrm{s}^{-1}$ ) ont donc été retenues pour suivre les variations de la viscosité Newtonienne des deux PET dans des ambiances d'oxygène représentatives des conditions réelles d'extrusion, c'est-à-dire :

- À pression partielle d'oxygène constante : $0 ; 0,6 ; 1 ; 9$ et $21 \%$ de la pression atmosphérique, pour reproduire les modifications macromoléculaires locales (prédominance de coupures ou de soudures de chaînes) à une position $z$ dans le réacteur d'extrusion.

- À pression partielle d'oxygène variable : alternances de $0 \%$ et $21 \%$ de la pression atmosphérique, pour reproduire l'historique des modifications macromoléculaires (successions de coupures et soudures de chaînes) pendant une opération complète d'extrusion.

\section{Résultats et discussion}

\subsection{Essais iso-atmosphériques}

Aux erreurs expérimentales près, on n'observe pas, au niveau rhéologique, de différences significatives entre les cinétiques de vieillissement thermique des deux lots de PET régénérés fournis par la société SOREPLA. C'est pourquoi, dans ce qui va suivre, on présentera les courbes de variations de la viscosité Newtonienne $\eta(t)$ normalisée par sa valeur initiale $\eta(t=0)$, pour s'affranchir des différences initiales entre les PET 1 et PET 2, et on ne fera plus de distinction entre ces deux polymères.

Des exemples de courbes de variation de la viscosité Newtonienne $\eta$ dans diverses ambiances d'oxygène sont présentés sur la figure 6 . On observe clairement une dépendance du comportement rhéologique du PET avec la pression partielle d'oxygène :

- comme on s'y attendait, en absence d'oxygène $(0 \%$ de la pression atmosphérique), le PET est relativement stable à $280^{\circ} \mathrm{C}$. En effet, après une légère diminution, qui peut être raisonnablement attribuée à la décomposition thermique des principales irrégularités structurales du PET, à savoir, les unités diéthylène glycol, $\eta$ atteint une valeur asymptotique ;

- aux plus faibles pressions partielles d'oxygène (typiquement $P_{\mathrm{O}_{2}}<9 \%$ de la pression atmosphérique), $\eta$ augmente lentement jusqu'à gélification complète du polymère. Ainsi, les soudures l'emportent sur les coupures de chaînes. D'après l'équation (19), on est dans la situation où : $4 \mathrm{X}>\mathrm{S}$;

- à la pression critique $P_{C}=9 \%$ de la pression atmosphérique, les valeurs de $\eta$ restent à peu près constantes. Les soudures équilibrent donc les coupures de chaînes et $l^{\prime}$ on a $: S=4 X$;

- aux plus fortes pressions partielles d'oxygène $\left(P_{\mathrm{O}_{2}}>P_{\mathrm{C}}\right), \eta$ diminue brutalement. Les coupures l'emportent donc largement sur les soudures de chaînes et l'on a : $\mathrm{S}>4 \mathrm{X}$. 


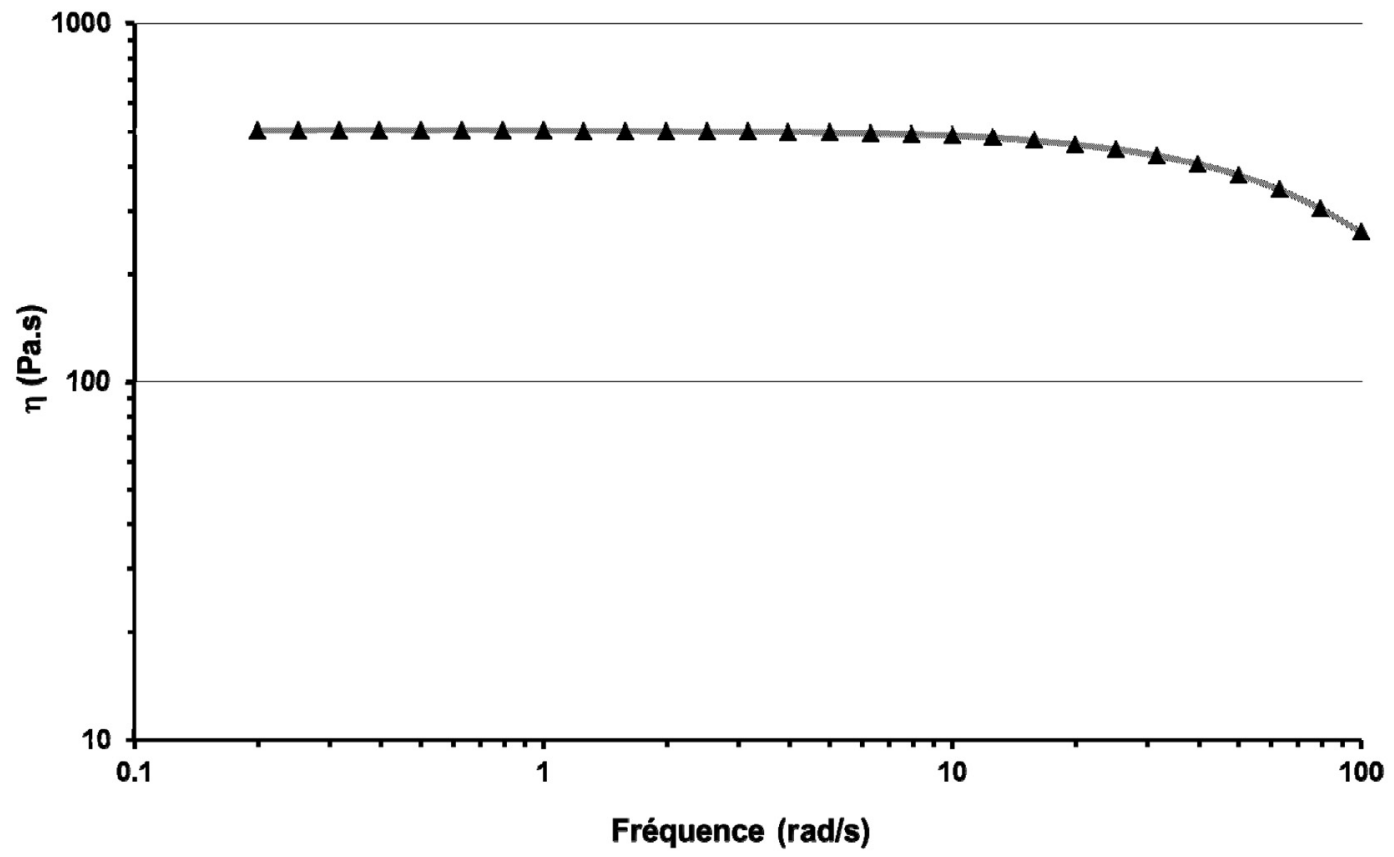

Fig. 5. Comportement rhéologique du PET 2 initial à $280^{\circ} \mathrm{C}$. Partie réelle de la viscosité dynamique en fonction de la fréquence angulaire.

Fig. 5. Rheological behavior of as received PET 2 at $280^{\circ} \mathrm{C}$. Real part of dynamic viscosity versus angular frequency.

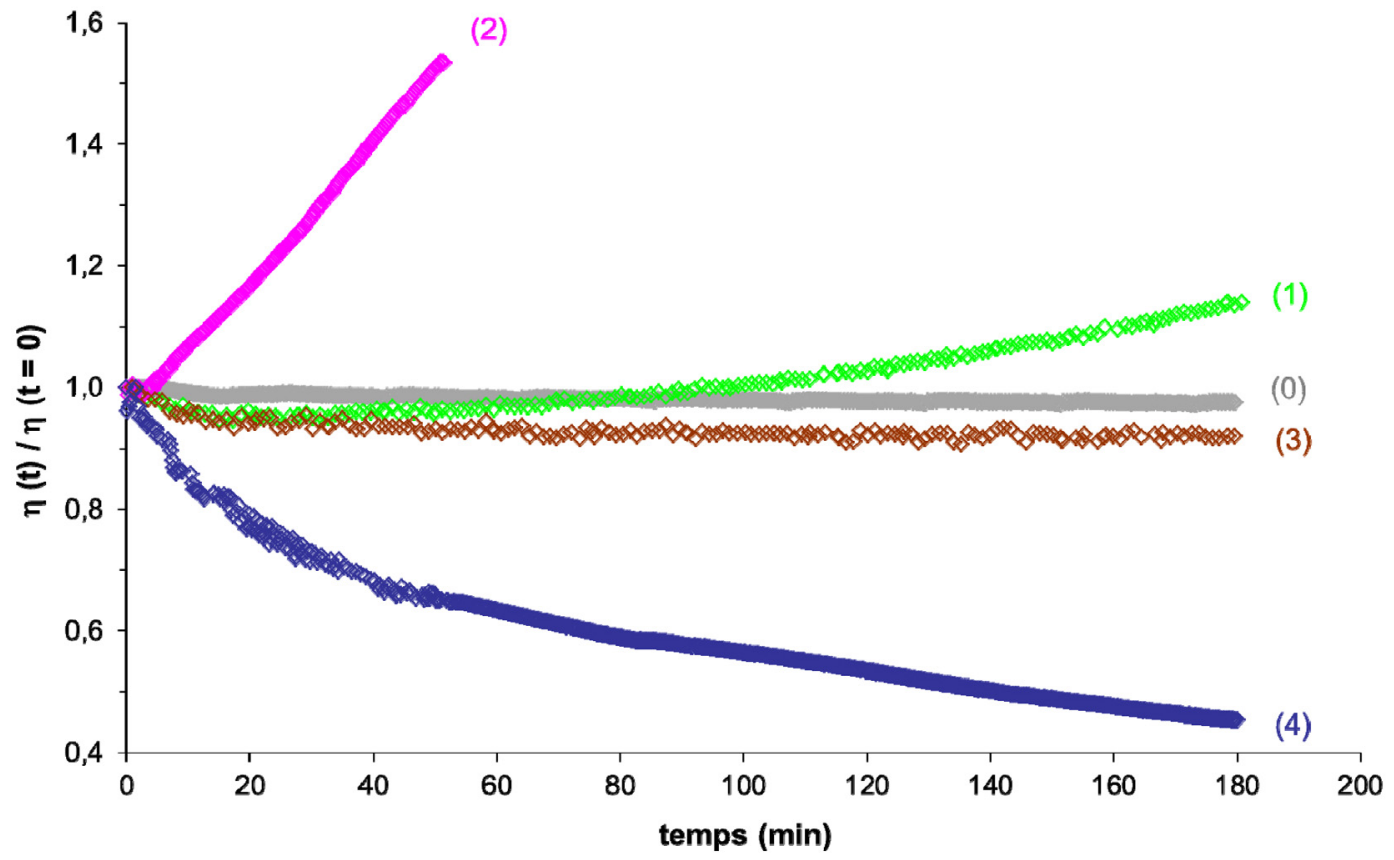

Fig. 6. Variations de la viscosité Newtonienne à $280^{\circ} \mathrm{C}$ à diverses pressions partielles d'oxygène : $0 \%(0) ; 0,6 \%(1) ; 1 \%(2) ; 9 \%(3)$ et $21 \%$ de la pression atmosphérique (4).

Fig. 6. Changes in Newtonian viscosity at $280{ }^{\circ} \mathrm{C}$ under various oxygen partial pressures: $0 \%(0) ; 0.6 \%$ (1); $1 \%$ (2); $9 \%$ (3) and $21 \%$ of atmospheric pressure (4). 


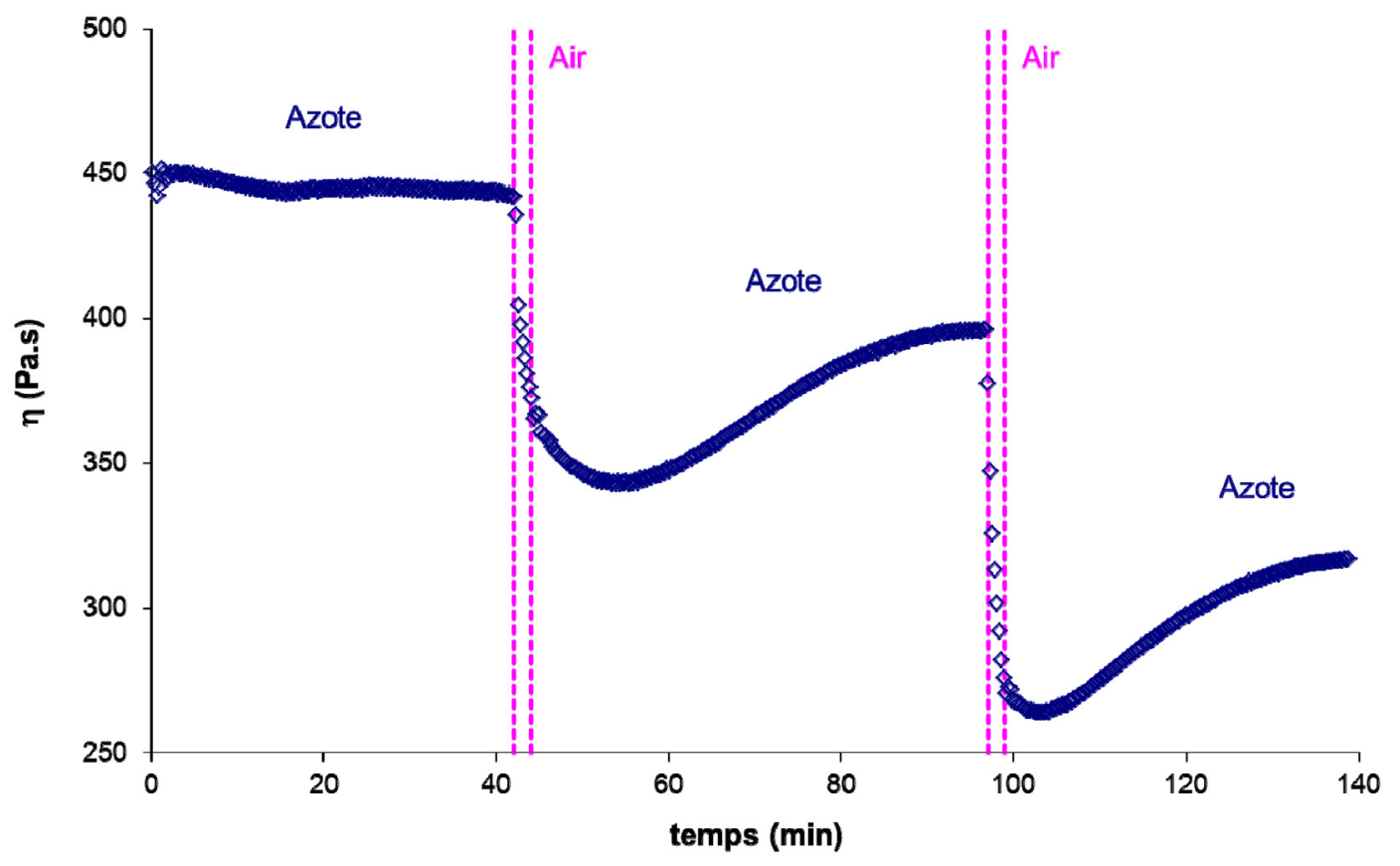

Fig. 7. Variations de la viscosité Newtonienne à $280^{\circ} \mathrm{C}$ pendant deux alternances azote/air à pression atmosphérique. Durée des expositions successives sous azote : 42, 53 puis $40 \mathrm{~min}$. Durée des expositions sous air : 2 min dans les deux cas.

Fig. 7. Changes in Newtonian viscosity at $280{ }^{\circ} \mathrm{C}$ during two nitrogen/air alternations under atmospheric pressure. Duration of successive exposures under nitrogen: 42, 53 then 40 min. Duration of exposures under air: 2 min in both cases.

En portant la valeur de $P_{C}$ dans l'équation (4), il est possible de calculer la concentration d'oxygène critique délimitant les régions «fortement oxygénées » de la région «sous-oxygénée» dans une extrudeuse. L'application numérique conduit à :

$$
\left[\mathrm{O}_{2}\right]\left(z_{1}\right)=\left[\mathrm{O}_{2}\right]\left(z_{2}\right)=\left[\mathrm{O}_{2}\right]_{\mathrm{C}}=4,2 \times 10^{-4} \mathrm{~mol} \cdot \mathrm{l}^{-1} \text {. }
$$

\subsection{Essais séquentiels}

Un exemple de courbe de variation de la viscosité Newtonienne $\eta$ du PET 1 au cours deux alternances azote/air à pression atmosphérique est présenté sur la figure 7 . On distingue trois différents domaines de variation de $\eta$. Par ordre chronologique :

- le premier domaine correspond à une phase de «stabilisation» des valeurs de $\eta$ sous azote. Comme vu précédemment, après une légère diminution, qui peut être raisonnablement attribuée à la décomposition thermique des principales irrégularités structurale du PET, $\eta$ atteint une valeur asymptotique ;

- le second domaine correspond à une chute catastrophique de $\eta$ dans l'air. Dans ce domaine, on a remplacé l'azote par l'air ambiant, pendant environ $2 \mathrm{~min}$, dans la cavité du rhéomètre. Les coupures l'emportent donc largement sur les soudures de chaînes. On note l'absence de période d'induction, c'est-à-dire que la vitesse de décroissance de $\eta$ est maximale dès l'introduction de l'air dans la cavité. Il s'agit là d'un argument fort en faveur de la présence d'un mécanisme d'amorçage extrinsèque d'oxydation. La réaction directe de l'oxygène moléculaire avec le polymère (1o) devrait permettre de rendre compte, sans l'emploi d'hypothèse ou de paramètre ajustable supplémentaire, de cette importante caractéristique de la cinétique d'oxydation du PET à l'état fondu à $280^{\circ} \mathrm{C}$;

- le troisième domaine correspond une variation non monotone de $\eta$ lorsque la pression partielle d'oxygène baisse de $21 \%$ à $0 \%$ de la pression atmosphérique. Dans ce domaine, on a remplacé l'air ambiant par l'azote dans la cavité du rhéomètre, jusqu'à stabilisation des valeurs de $\eta$. On observe, tour à tour, un ralentissement de la chute de $\eta$, amorcée sous air, et le passage par un minimum de $\eta$ vraisemblablement lorsque la 
pression partielle d'oxygène atteint $9 \%$ de la pression atmosphérique. C'est, en effet, à cette valeur critique que les soudures équilibrent les coupures de chaînes (voir Fig. 6). Dès que la pression partielle d'oxygène devient inférieure à cette valeur critique, les soudures l'emportent sur les coupures de chaînes, et l'on observe une ré-augmentation de $\eta$. Cette augmentation cesse quand l'oxygène est totalement consommé dans la cavité du rhéomètre.

\subsection{Modélisation cinétique}

Nous avons utilisé le modèle cinétique, constitué des équations (8) à (20), pour simuler les courbes de variation de $\eta$ des figures 6 et 7 . Les valeurs des concentrations initiales, des constantes de vitesse et des rendements sont rapportées dans les tableaux 3 et 4 . Ces valeurs appellent les commentaires suivants :

(i) la concentration initiale en irrégularités structurales est tout à fait raisonnable. Elle représente environ 1,7 \% en moles des unités monomères, ce qui est en parfait accord avec la littérature [23] ;

(ii) les constantes de vitesse $k_{1 u}$ à $k_{63}$, excepté $k_{4}$, ont été déterminées dans une étude antérieure pour le PE [49]. Des valeurs de $k_{4}$ supérieures à $10^{12} 1 \mathrm{~mol}^{-1} \mathrm{~s}^{-1}$ sont, a priori, difficiles à expliquer pour des espèces aussi rares que les radicaux alkyl. Cependant, on s'attend à ce que, à haute température, à l'état fondu, la réaction de transfert d'hydrogène par migration de valence joue un rôle crucial :

$$
\mathrm{P}^{\circ}+\mathrm{PH} \rightarrow \mathrm{PH}+\mathrm{P}^{\circ} .
$$

(iii) Les constantes de vitesse $k_{10}, k_{0 i}$ et $k_{7}$, ainsi que les rendements $\gamma_{1}$ et $\gamma_{4}$, ont été déterminés dans cette étude. La valeur de la constante $k_{10}$ est suffisamment élevée pour que, à $280^{\circ} \mathrm{C}$, le caractère en «boucle fermée » du mécanisme d'oxydation soit totalement perdu. En effet, on se trouve dans la situation où les vitesses initiales de production de radicaux par les réactions $(1 \mathrm{o})$ et $(1 \mathrm{u})$ sont du même ordre de grandeur. À titre d'exemple, dans l'air à pression atmosphérique, ces vitesses s'écrivent :

$v_{1 o}=4 k_{1 o}[\mathrm{PH}]_{0}\left[\mathrm{O}_{2}\right] \approx 4,9 \times 10^{-5} \mathrm{moll}^{-1} \mathrm{~s}^{-1}$ et

$$
v_{1 u}=k_{1 u}[\mathrm{POOH}]_{0} \approx 5,0 \times 10^{-5} \mathrm{moll}^{-1} \mathrm{~s}^{-1}
$$

soit :

$$
v_{10} \approx v_{1 u}
$$

Par contre, la concentration initiale en acides carboxyliques et la valeur de la constante $k_{7}$ sont trop faibles pour que la réaction d'allongement de chaînes, par condensation des paires d'acide carboxylique, puisse significativement impacter le comportement rhéologique du polymère à l'état fondu, dans l'échelle de temps d'une succession d'opérations d'extrusion. Mais, à plus long terme, quand les acides carboxyliques se seront accumulés dans le milieu réactionnel, il est possible que cette réaction joue enfin un rôle important.

Ainsi, en dessous de la pression critique d'oxygène ( $9 \%$ de la pression atmosphérique), les branchements de chaines sont la principale source de problèmes pendant le recyclage mécanique du PET par extrusion. Le rendement $\gamma_{4}$ de la réticulation des radicaux alkyl est de $1^{\prime}$ ordre de $17 \%$ à $280^{\circ} \mathrm{C}$. Cette réaction est en compétition avec la réaction de dismutation :
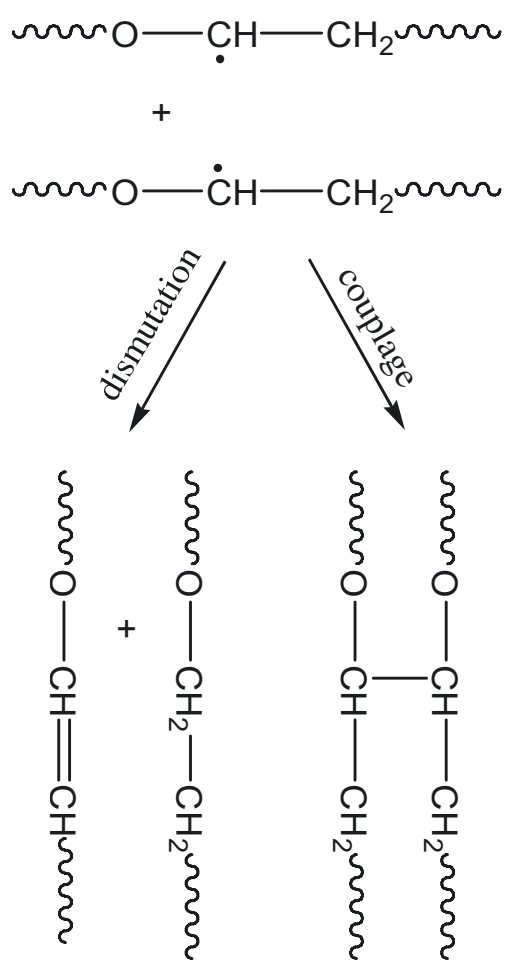

En revanche, au-dessus de cette pression critique, les coupures de chaînes sont la principale source de problèmes pendant le recyclage mécanique du PET par extrusion. Le 
Tableau 3. Concentrations initiales (en $\mathrm{moll}^{-1}$ ) des différentes espèces réactives.

Table 3. Initial concentrations (in mol $l^{-1}$ ) of the different reactive species.

\begin{tabular}{cc}
\hline Concentration & Valeur \\
\hline$[\text { Irreg }]_{0}$ & $2,4 \times 10^{-3}$ \\
{$[\mathrm{POOH}]_{0}$} & $10^{-4}$ \\
{$[\mathrm{PH}]_{0}$} & 14 \\
{$[\mathrm{POH}=\mathrm{O}]_{0}$} & $2,1-2,4 \times 10^{-2}$ \\
\hline
\end{tabular}

rendement $\gamma_{1}$ de la coupure $\beta$ des radicaux alkoxy est de $l^{\prime}$ ordre de $84 \%$ à $280^{\circ} \mathrm{C}$. Cette réaction est en compétition avec la réaction d'arrachement d'hydrogène :
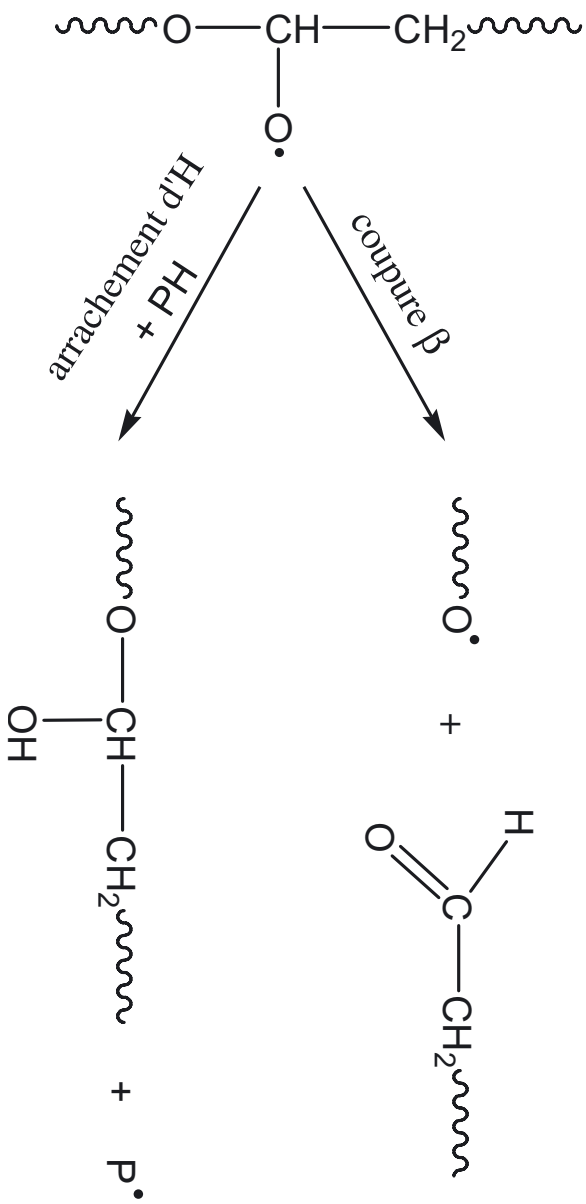

Des exemples de simulation des variations de $\eta$ sont rapportés sur les figures 8 et 9 . On constate que le modèle cinétique décrit de manière satisfaisante l'ensemble des résultats expérimentaux, aussi bien aux pressions partielles d'oxygène constantes, qu'aux pressions partielles d'oxygène variables. On peut donc considérer que la validité du modèle cinétique a été vérifiée.

Comme envisagé dans la section précédente, à très long terme, les allongements de chaînes affectent, à leur tour, le comportement rhéologique du PET (Fig. 10). Ainsi, après environ $275 \mathrm{~min}$ d'exposition à $280^{\circ} \mathrm{C}$
Tableau 4. Paramètres utilisés pour la modélisation cinétique à $280^{\circ} \mathrm{C}$.

Table 4. Parameters used for kinetic modeling at $280^{\circ} \mathrm{C}$.

\begin{tabular}{ccc}
\hline Paramètre & Valeur & Unité \\
\hline$k_{i}$ & $7,1 \times 10^{-4}$ & $\mathrm{~s}^{-1}$ \\
$k_{1 o}$ & $9 \times 10^{-4}$ & $1 \mathrm{~mol}^{-1} \mathrm{~s}^{-1}$ \\
$k_{1 u}$ & $5 \times 10^{-1}$ & $\mathrm{~s}^{-1}$ \\
$k_{2}$ & $10^{8}$ & $1 \mathrm{~mol}^{-1} \mathrm{~s}^{-1}$ \\
$k_{3}$ & $1,9 \times 10^{3}$ & $1 . \mathrm{mol}^{-1} \mathrm{~s}^{-1}$ \\
$k_{4}$ & $6 \times 10^{13}$ & $1 \mathrm{~mol}^{-1} \mathrm{~s}^{-1}$ \\
$k_{5}$ & $4 \times 10^{11}$ & $1 \mathrm{~mol}^{-1} \mathrm{~s}^{-1}$ \\
$k_{60}$ & $10^{10}$ & $1 \mathrm{~mol}^{-1} \mathrm{~s}^{-1}$ \\
$k_{62}$ & $4 \times 10^{5}$ & $\mathrm{~s}^{-1}$ \\
$k_{63}$ & $10^{8}$ & $\mathrm{~s}^{-1}$ \\
$k_{7}$ & $1,7 \times 10^{-6}$ & $1 \mathrm{~mol}^{-1} \mathrm{~s}^{-1}$ \\
$\gamma_{1}$ & 83,9 & $\%$ \\
$\gamma_{4}$ & 17,4 & $\%$ \\
\hline
\end{tabular}

du PET dans l'air ambiant, on observe une ré-augmentation de $\eta$, sans doute jusqu'à gélification (réticulation) du polymère. On peut constater, sur la figure 10, que le modèle cinétique rend parfaitement compte de cette évolution, ce qui confirme sa bonne qualité prédictive.

\section{Conclusions}

Pendant son recyclage mécanique par extrusion, le PET subit des modifications réversibles de sa structure macromoléculaire induites par des réactions d'hydrolyse, d'estérification et de transestérification. Mais, il subit aussi des modifications macromoléculaires irréversibles supposées être, a priori, induites par une grande variété de processus chimiques (radicalaires, mais aussi ioniques).

Comme une extrudeuse est un réacteur relativement complexe (existence de gradients de concentration d'oxygène, de température, et de cisaillement), nous avons choisi la cavité d'un rhéomètre pour étudier ces modifications macromoléculaires et leur impact sur les propriétés rhéologiques, dans des conditions d'exposition soigneusement contrôlées.

Ces essais ont permis d'établir que, dans l'échelle de temps d'une succession d'opérations d'extrusion, la thermo-oxydation du polymère est de loin la principale source de problèmes. Les coupures de chaînes prédominent dans les zones «fortement oxygénées » (l'alimentation et la tête), alors que les soudures de chaînes prédominent dans la zone «sous-oxygénée » (au centre) du réacteur d'extrudeuse. Ainsi, au bilan, la prédominance relative de ces deux types de 


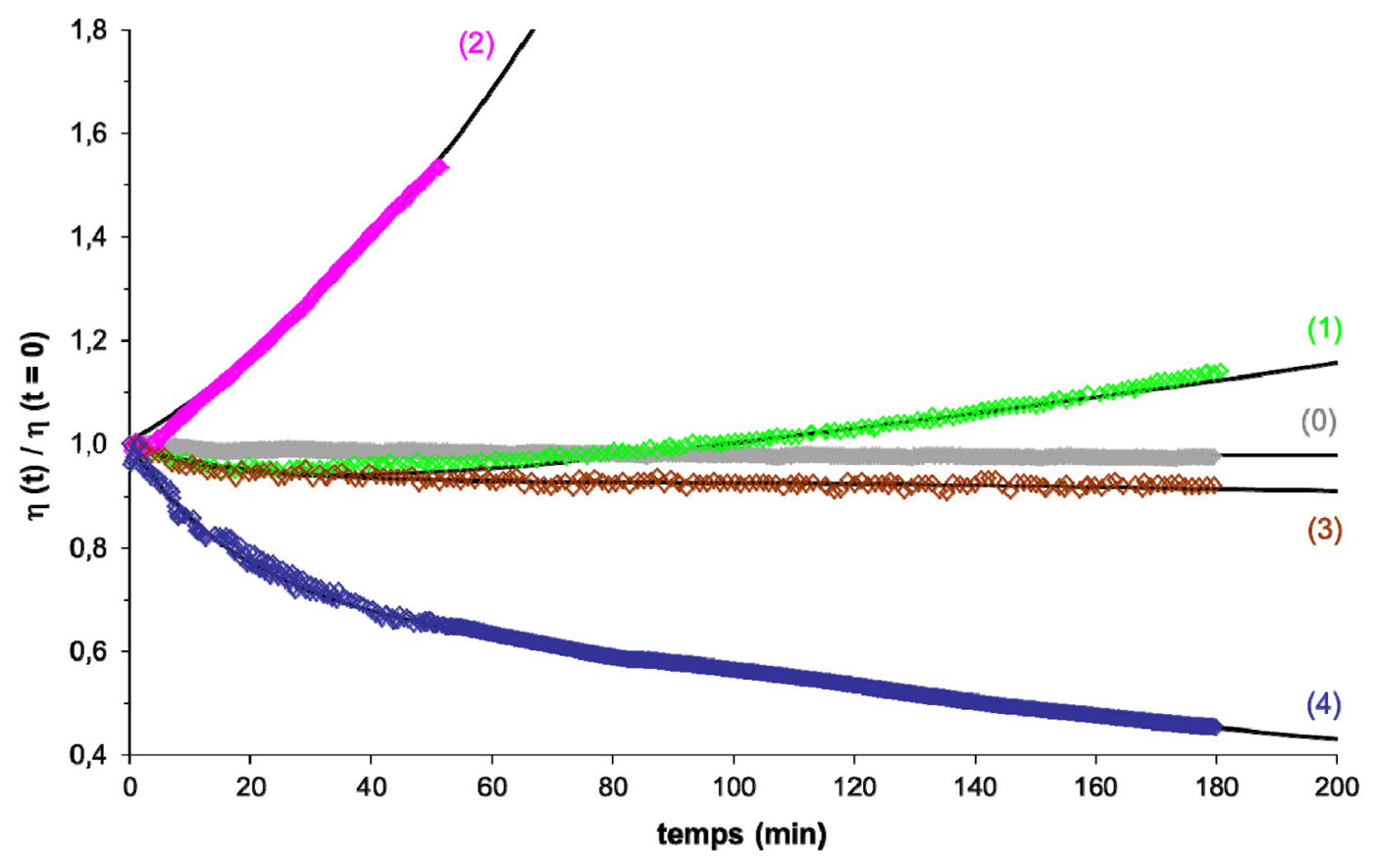

Fig. 8. Simulation des variations de la viscosité Newtonienne à $280^{\circ} \mathrm{C}$ à diverses pressions partielles d'oxygène : $0 \%(0) ; 0,6 \%(1) ; 1 \%(2) ; 9 \%(3)$ et $21 \%$ de la pression atmosphérique (4). Points : données expérimentales. Courbe : modèle cinétique.

Fig. 8. Simulations of the changes in Newtonian viscosity at $280{ }^{\circ} \mathrm{C}$ under various oxygen partial pressures: $0 \%(0) ; 0.6 \%$ (1); $1 \%$ (2); $9 \%$ (3) and $21 \%$ of atmospheric pressure (4). Dots: experimental data. Curves: kinetic model.

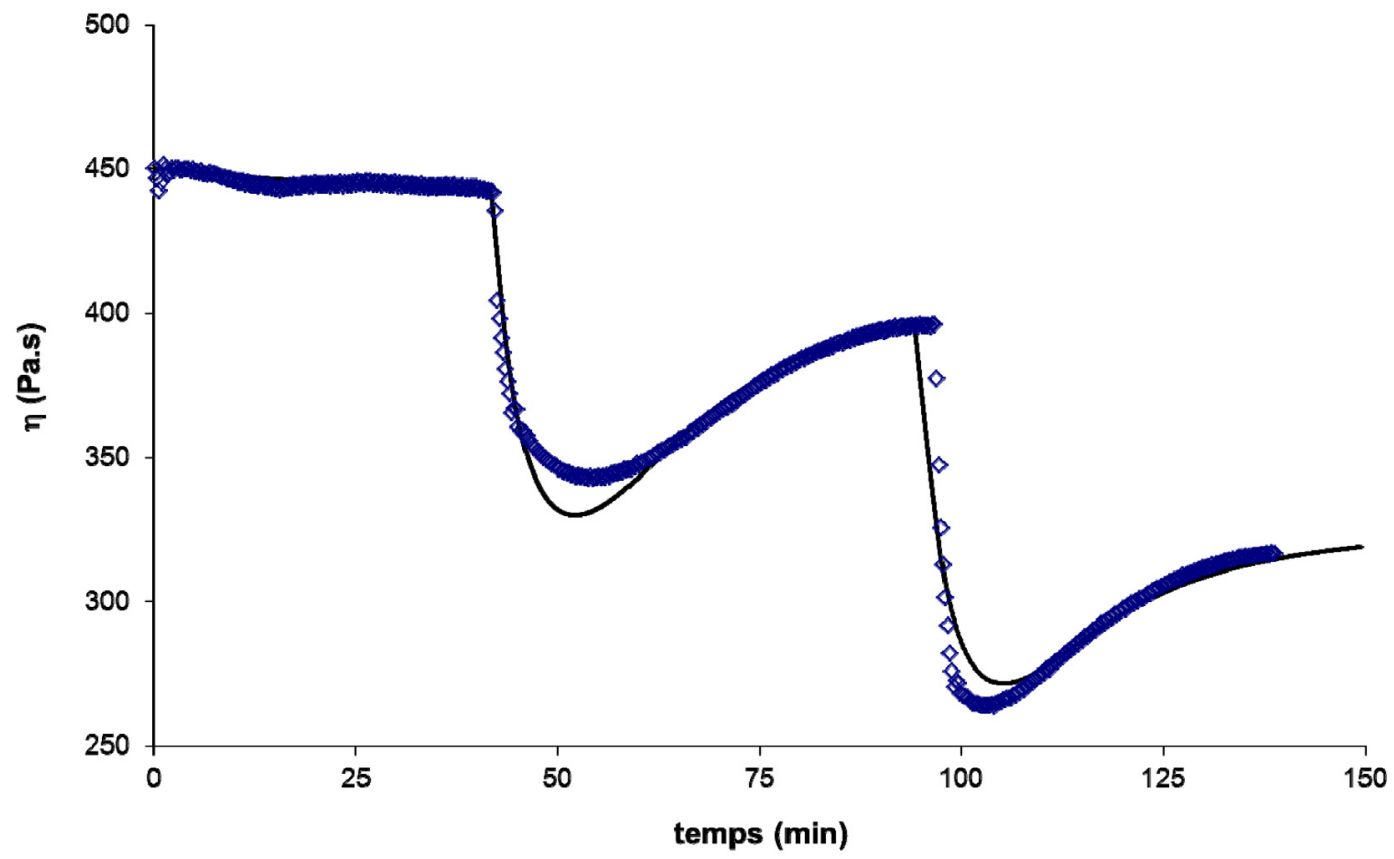

Fig. 9. Simulation des variations de la viscosité Newtonienne à $280{ }^{\circ} \mathrm{C}$ pendant deux alternances azote/air à pression atmosphérique. Points : données expérimentales. Courbe : modèle cinétique. Fig. 9. Simulation of the changes in Newtonian viscosity at $280^{\circ} \mathrm{C}$ during two nitrogen/air alternations under atmospheric pressure. Dots: experimental data. Curve: kinetic model. 


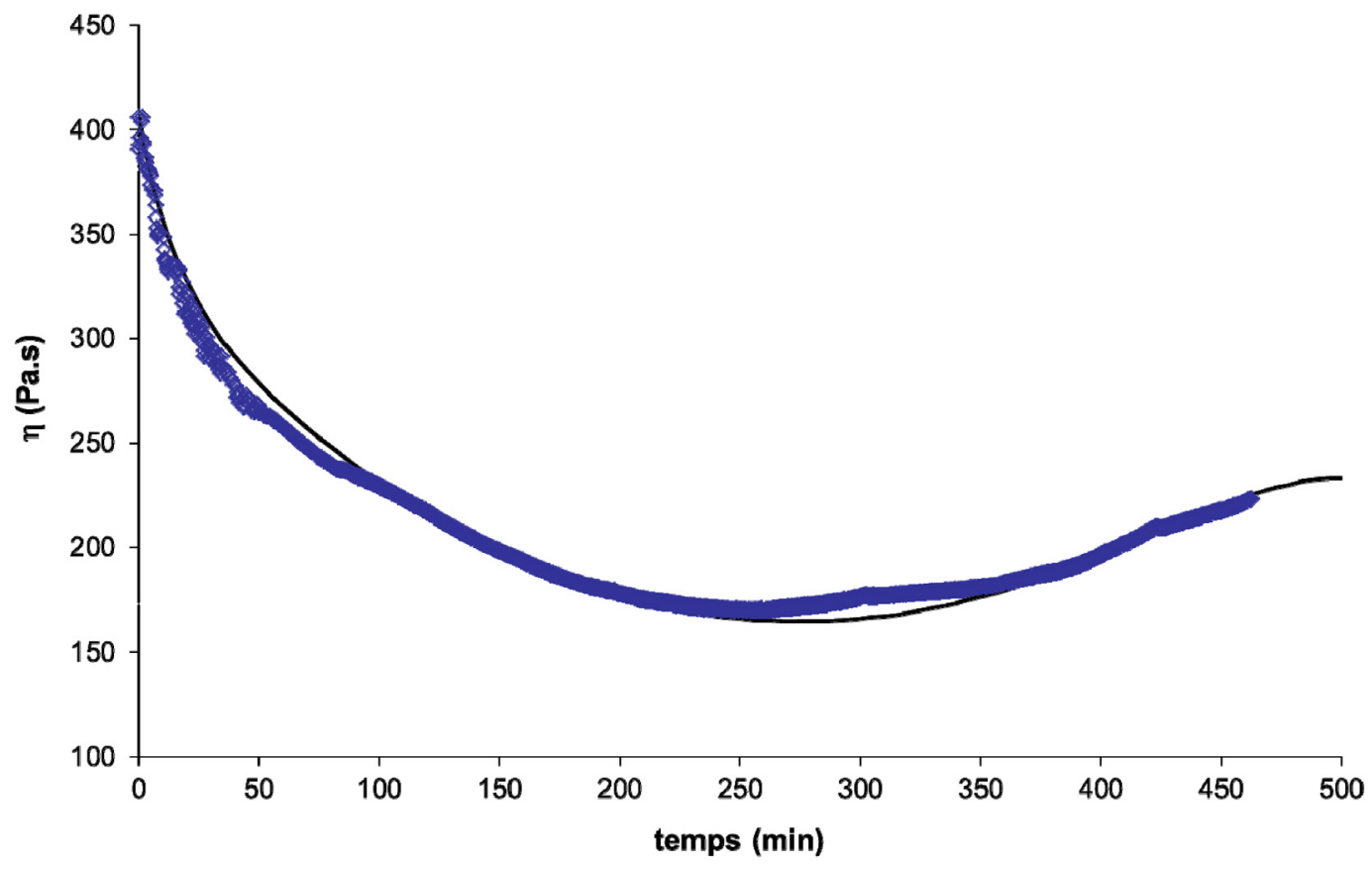

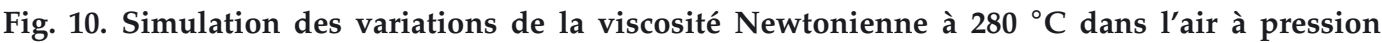
atmosphérique. Points : données expérimentales. Courbe : modèle cinétique.

Fig. 10. Simulation of the changes in Newtonian viscosity at $280^{\circ} \mathrm{C}$ under atmospheric air. Dots: experimental data. Curves: kinetic model.

modifications macromoléculaires est étroitement liée à la géométrie et aux dimensions de l'extrudeuse (en particulier, à l'aire des sections d'alimentation et de tête, et la longueur de la vis), ce qui explique la grande disparité des évolutions de comportements rhéologiques rapportées dans la littérature. Dans l'échelle de temps de quelques opérations d'extrusion, les soudures de chaînes consistent essentiellement à des branchements de chaînes. Mais à plus long terme, les allongements de chaînes peuvent jouer, à leur tour, un rôle majeur.

En parallèle, nous avons dérivé, du schéma d'oxydation en "boucle fermée » établi pour un substrat poly(méthylénique) dans une étude antérieure [49], un modèle cinétique de vieillissement thermique du PET à haute température, à l'état fondu, pour vérifier les hypothèses précédentes. Ce modèle décrit, de manière satisfaisante, l'ensemble des résultats rhéométriques obtenus à $280^{\circ} \mathrm{C}$ à des pressions partielles d'oxygène constantes, mais aussi à des pressions partielles d'oxygène variables (entre $0 \%$ et $21 \%$ de la pression atmosphérique).

\section{Références}

[1] R.D. Leaversuch, Modern Plastics 68 (1991) $40-43$
[2] A. Launay, F. Thominette, J. Verdu, Polym. Degrad. Stab. 46 (1994) 319-324

[3] H. Zimmermann, Degradation and Stabilization of Polyesters, in: Developments in Polymer Degradation, Vol. 5, edited by N. Grassie, Applied Science Publishers, London, New York, Chap. 3, pp. 79-119, 1984

[4] B. Culbert, A. Christel, Continuous solidstate polycondensation of polyesters, in: Modern Polyesters: Chemistry and Technology of Polyesters and Copolyesters, edited by J. Scheirs, T.E. Long, John Wiley \& Sons Ltd (Wiley series in Polymer Science), Sussex, England, pp. 143-194, 2003.

[5] E.P. Goodings, J. Soc. Chem. Ind. (London), Monograph 13 (1961) 211-228

[6] L.H. Buxbaum, Ang. Chem. Int. Ed. 7 (1968) 182-190

[7] H. Zimmerman, N.T. Kim, Polym. Eng. Sci. 20 (1980) 680-683

[8] K.C. Khemani, Polym. Degrad. Stab. 67 (2000) 91-99

[9] K. Yoda, A. Tsuboi, M. Wada, R. Yamareda, J. Appl. Polym. Sci. 14 (1970) 2357-2376

[10] I.C. McNeil, M. Bounekhel, Polym. Degrad. Stab. 34 (1991) 187-204

[11] H. Pohl, J. Am. Chem. Soc. 73 (1951) 5660-5661

[12] G. Montaudo, C. Puglisi, F. Samperi, Polym. Degrad. Stab. 42 (1993) 13-28

[13] F. Villain, J. Coudane, M. Vert, Polym. Degrad. Stab. 43 (1994) 431-440

[14] F. Samperi, C. Puglisi, R. Alicata, G. Montaudo, Polym. Degrad. Stab. 83 (2004) 310 
[15] M. Edge, N.S. Allen, R. Wiles, W.A. McDonald, S.V. Mortlock, Polymer 36 (1995) 227-234

[16] M. Edge, R. Wiles, N.S. Allen, W.A. McDonald, S.V. Mortlock, Polym. Degrad. Stab. 53 (1996) 141-151

[17] B.M. Kovarskaya, I.I. Levanstovskaya, A.B. Blumenfeld, G.V. Draluk, Plasticheskie Massy 5 (1968) 42-46

[18] I. Marshall, A. Todd, Trans. Faraday Soc. 49 (1953) 67-78

[19] C.F.L. Ciolacu, N.R. Choudhury, N.K. Dutta, N.H. Voelcker, Macromolecules 39 (2006) 7872-7881

[20] B.J. Holland, J.N. Hay, Polymer 43 (2002) 1797-1804

[21] G. Botelho, A. Queiros, P. Gijsman, Polym. Degrad. Stab. 74 (2001) 39-48

[22] G.W. Halek, J. Polym. Sci.: Polym. Symp. 74 (1986) 83-92

[23] W.A. MacDonald, Polym. Int. 51 (2002) 923930

[24] W. Romao, M.F. Franco, Y.E. Corilo, M.N. Eberlin, M.A.S. Spinacé, M.-A. De Paoli, Polym. Degrad. Stab. 94 (2009) 1849-1859

[25] H.A. Lecomte, J.J. Ligat, Polym. Degrad. Stab. 91 (2006) 681-689

[26] C.F.L. Ciolacu, N.R. Choudhury, N.K. Dutta, Polym. Degrad. Stab. 91 (2006) 875-885

[27] G. Botelho, A. Queiros, P. Gijsman, Polym. Degrad. Stab. 67 (2000) 13-20

[28] J. Yang, Z. Xia, F. Kong, X. Ma, Polym. Degrad. Stab. 95 (2010) 53-58

[29] J. Scheirs, Polymer Recycling: Science, Technology and Application, John Wiley \& Sons Ltd (Wiley series in Polymer Science), Sussex, England, Chap. 4, pp. 120-182, 1998

[30] X. Colin, J. Verdu, C.R. Chim. Acad. Sci. Chim. 9 (2006) 1380-1390

[31] F.P. La Mantia, M. Vinci, Polym. Degrad. Stab. 45 (1994) 121-125

[32] M. Paci, F.P. La Mantia, Polym. Degrad. Stab. 61 (1998) 417-420

[33] M. Frounchi, Macromol. Symp. 144 (1999) 465-469

[34] M.A.S. Spinacé, M.-A. De Paoli, J. Appl. Polym. Sci. 80 (2001) 20-25
[35] R. Assadi, X. Colin, J. Verdu, Polymer 45 (2004) 4403-4412

[36] K.L. Nait-Ali, X. Colin, A. Bergeret, L. Ferry, P. Ienny, Rheological modelisation of PET degradation during its recycling by extrusion, in: Proceedings of 23rd Annual Meeting of Polymer Processing Society (PPS-23), CDRom, 8 p., 2007

[37] J.D. Badia, F. Vilaplana, S. Karlsson, A. Ribes-Greus, Polym. Test. 28 (2009) 169-175

[38] A. Oromiehie, A. Mamizadeh, Polym. Int. 53 (2004) 728-732

[39] S.D. Mancini, M. Zanin, Mater. Res. 2 (1999) 33-38

[40] N. Torres, J.-J. Robin, B. Boutevin, Eur. Polym. J. 36 (2000) 2075-2080

[41] S.D. Mancini, M. Zanin, J. Appl. Polym. Sci. 76 (2000) 266-275

[42] S.A. Jabarin, E.A. Lofgren, Polym. Eng. Sci. 24 (1984) 1056-1063

[43] F. Villain, J. Coudane, M. Vert, J. Appl. Polym. Sci. 52 (1994) 55-60

[44] B. Fayolle, E. Richaud, X. Colin, J. Verdu, J. Mater. Sci. 43 (2008) 6999-7012

[45] H. Sobue, J. Kajiura, K.K. Zasshi, Ind. Chem. Sect. 62 (1959) 1766

[46] L. Audouin, V. Langlois, J. Verdu, J.C.M. De Bruijn, J. Mater. Sci. 29 (1994) 569-583

[47] D.W. Van Krevelen, P.J. Hoftyzer, Properties of Polymers. Their Estimation and Correlation with Chemical Structure, $2^{\mathrm{e}}$ édition, Elsevier, Amsterdam, Chap. 18, p. 406,1976

[48] V. Stannett, Simples gases, in: Diffusion in Polymers, edited by J. Crank, G.S. Park, Academic Press, London, Chap. 2, pp. 4650, 1968

[49] X. Colin, L. Audouin, J. Verdu, M. RozentalEvesque, B. Rabaud, F. Martin, F. Bourgine, Polym. Eng. Sci. 49 (2009) 1429-1437

[50] O. Saito, J. Phys. Soc. Jpn 13 (1958) 198-206; J. Phys. Soc. Jpn 13, (1958) 1451-1464

[51] F. Bueche, J. Chem. Phys. 22 (1954) 603-609

[52] F. Bueche, J. Chem. Phys. 25 (1956) 599-601 